\title{
Spectrin is a mechanoresponsive protein shaping the architecture of intercellular invasion
}

Rui Duan ${ }^{1 \#^{\dagger}}$, Ji Hoon Kim ${ }^{1 \#}$, Khurts Shilagardi ${ }^{1 \#}$, Eric Schiffhauer ${ }^{2}$, Sungmin Son ${ }^{3}$, Donghoon

Lee $^{4}$, Shuo $\mathrm{Li}^{1}$, Graham Thomas ${ }^{5}$, Tianzhi Luo ${ }^{6}$, Daniel A. Fletcher ${ }^{3}$, Douglas N. Robinson ${ }^{2}$, and Elizabeth H. Chen ${ }^{1,4}$

${ }^{1}$ Department of Molecular Biology and Genetics, ${ }^{2}$ Department of Cell Biology, Johns Hopkins University School of Medicine, Baltimore, MD 21205, USA

${ }^{3}$ Department of Bioengineering, University of California, Berkeley, Berkeley, CA 94720, USA

${ }^{4}$ Department of Molecular Biology, UT Southwestern Medical Center, Dallas, TX 75390, USA

${ }^{5}$ Departments of Biology and of Biochemistry and Molecular Biology, Penn State University, University Park, PA 16802

${ }^{6}$ Department of Modern Mechanics, University of Science and Technology of China, Hefei, China

${ }^{\#}$ These authors contributed equally

${ }^{\dagger}$ Present address: Laboratory of Regenerative Medicine in Sports Science, School of Sports

Science, South China Normal University, Guangzhou, China 
Spectrin is a membrane skeletal protein best known for its structural role in maintaining cell shape and protecting cells from mechanical damage ${ }^{1-3}$. Here, we report that spectrin dynamically accumulates and dissolves at the fusogenic synapse, where an attacking fusion partner mechanically invades its receiving partner with actin-propelled protrusions to promote cell-cell fusion ${ }^{4-7}$. Using genetics, cell biology, biophysics and mathematical modeling, we demonstrate that unlike myosin II that responds to dilation deformation, spectrin exhibits a mechanosensitive accumulation in response to shear deformation, which is highly elevated at the fusogenic synapse. The accumulated spectrin forms an uneven network, which functions as a "sieve" to constrict the invasive fingerlike protrusions, thus putting the fusogenic synapse under high mechanical tension to promote cell membrane fusion. Taken together, our study has revealed a previously unrecognized function of spectrin as a dynamic mechanoresponsive protein that shapes the architecture of intercellular invasion. These findings have general implications for understanding spectrin function in other dynamic cellular processes beyond cell-cell fusion.

The mechanical properties of cells are dynamically controlled in many cellular processes, such as cell division, fusion, migration, invasion, and shape change. Spectrin is best known as a membrane skeletal protein critical for maintaining cell shape and providing mechanical support for plasma membrane ${ }^{1-3}$. In erythrocytes and neurons, spectrins, together with actin, ankyrin and associated proteins, form a static polygonal lattice structure $\mathrm{s}^{8-10}$ or an ordered periodic longitudinal array ${ }^{11}$ underneath the plasma membrane to protect cells from mechanical damage $^{12}$. Such a mechanoprotective function of spectrin is made possible by holding the spectrin network under constitutive tension ${ }^{13}$. However, in many cellular processes, mechanical tension is generated upon transient cell-cell interactions. How spectrins, which are expressed in 
most eukaryotic cells, respond to transient and localized mechanical stimuli in dynamic cellular processes remains largely unknown.

Cell-cell fusion is a dynamic process that occurs in fertilization, immune response, bone resorption, placenta formation, and skeletal muscle development and regeneration ${ }^{14,15}$. It has been demonstrated in a variety of cell fusion events from Drosophila to mammals that cell fusion is an asymmetric process ${ }^{4-7,16}$. At the site of fusion, known as the fusogenic synapse, an attacking fusion partner invades its receiving fusion partner with actin-propelled membrane protrusions ${ }^{4-7,16}$, while the receiving fusion partner mounts a myosin II (Myoll)-mediated mechanosensory response ${ }^{6}$. The invading and resisting forces from the two fusion partners, in turn, push the two cell membranes to close proximity and put the fusogenic synapse under high mechanical tension to promote fusogen engagement and cell membrane merger ${ }^{5,6}$. Although previous studies have shown that multiple long (1-2 $\mu \mathrm{m})$ and thin ( 200 $\mathrm{nm}$ in diameter) invasive protrusions from the attacking fusion partner are required for cell-cell fusion ${ }^{4,5,17,18}$, it is unclear how these protrusions are spatially constricted and shaped in order to provide high mechanical tension at the fusogenic synapse.

In a genetic screen for new components required for cell-cell fusion, we have uncovered an essential function for spectrin in Drosophila myoblast fusion. Spectrins are composed of $\alpha$ - and $\beta$-subunits, which laterally associate to form antiparallel heterodimers and then assemble headto-head to form flexible, rod-like heterotetramers with actin-binding domains at the two ends ${ }^{1-3}$.

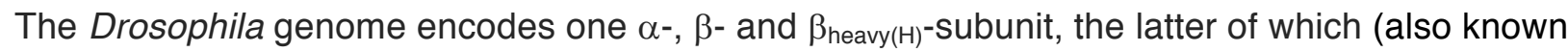
as karst) is homologous to $\beta$ V-spectrin in vertebrates ${ }^{19}$. Zygotic mutants of $\alpha$ - or $\beta_{\mathrm{H}^{-}}$-spectrin exhibited minor myoblast fusion defects (Fig. 1a-h, i) likely due to maternal contribution, 


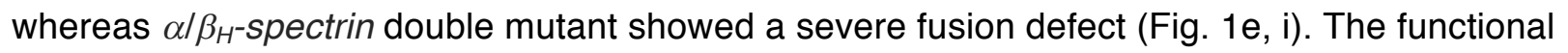
specificity of $\alpha / \beta_{H^{-}}$spectrin in myoblast fusion was demonstrated by a genetic rescue experiment, in which full-length $\beta_{\mathrm{H}}$-spectrin expressed in all muscle cells rescued the myoblast fusion defect in $\beta_{H}$-spectrin mutant embryos (Fig. 1f, i), and by an overexpression experiment, in which a dominant negative form of $\beta_{H^{-}}$spectrin (mini- $\beta_{H^{-}}$spectrin, deleting 15 of the 29 spectrin repeats ${ }^{20}$ expressed in muscle cells enhanced the fusion defect of $\beta_{H^{-}}$spectrin mutant embryos (Fig. 1g, i). Furthermore, expressing $\beta_{\mathrm{H}}$-spectrin specifically in the receiving fusion partners, the muscle founder cells, rescued the fusion defect (Fig. 1h, i), demonstrating that $\alpha / \beta_{\mathrm{H}^{-}}$spectrin functions specifically in the receiving cells.

To determine the subcellular localization of $\alpha / \beta_{\mathrm{H}^{-}}$-spectrin, we performed antibody-labeling experiments using anti- $\alpha$ - and $\beta_{\mathrm{H}^{-}}$-spectrin antibodies in wild-type embryos (Fig. 1j, k), and an anti-Flag antibody in a protein trap line, kst $^{\text {MIO3134-GFSTF.1 }}$, which carries 3xFlag-tagged $\beta_{\mathrm{H}^{-}}$Spectrin (Fig. 1l). Both $\alpha$ - and $\beta_{\mathrm{H}^{-}}$spectrin are highly enriched at the fusogenic synapse marked by an Factin-enriched focus, whereas no enrichment was observed for $\beta$-spectrin, which was expressed at a high level in epithelial cells (Extended Data Fig. 1a). The $\beta_{\mathrm{H}^{-}}$spectrin enrichment at the fusogenic synapse largely colocalized with that of Dumbfounded (Duf), an Ig domain-containing founder cell-specific adhesion molecule ${ }^{21}$ (Fig. 1j-I). Moreover, expressing a V5-tagged $\beta_{\mathrm{H}^{-}}$ spectrin specifically in founder cells led to the accumulation of $\beta_{\mathrm{H}^{-}}$spectrin-V5 at the fusogenic synapse (Fig. $1 \mathrm{~m})$. In contrast, expressing $\beta_{\mathrm{H}^{-}}$spectrin-V5 specifically in the attacking fusion partners, the fusion competent myoblasts (FCMs), did not result in its accumulation at the fusogenic synapse (Fig. 1n). These results further support the functional requirement for $\alpha / \beta_{\mathrm{H}^{-}}$ spectrin in founder cells. 
To investigate whether $\beta_{\mathrm{H}}$-spectrin forms a stable membrane skeletal network at the fusogenic synapse, we performed live imaging experiments in Drosophila embryos. Surprisingly, instead of forming a stable network, $\beta_{\mathrm{H}}$-spectrin exhibited dynamic accumulation and dissolution at the fusogenic synapse accompanying the appearance and disappearance of the actin-enriched invasive structure from the FCM, known as a podosome-like structure (PLS) ${ }^{4}$ (Supplementary Video 1; Extended Data Fig. 2a, b). Previous studies have shown that the density of an actin focus correlates with its invasiveness, such that loosely packed actin filaments are less invasive, as in $d p a k 3$ mutant embryos ${ }^{18}$. We found that the amount of $\beta_{\mathrm{H}^{-}}$spectrin accumulation correlated with the density of the F-actin foci, as shown in both wild-type (Supplementary Video 1;

Extended Data Fig. 2a, b) and dpak3 mutant embryos (Supplementary Video 2; Extended Data Fig. 2c, d). Specifically, $\beta_{\mathrm{H}^{-}}$spectrin accumulation in dpak3 mutant embryos was overall weaker than that in wild-type embryos, except for a few spectrin "hot spots" associated with the occasionally aggregated small F-actin foci (Extended Data Fig. 2c, d). These findings suggest that spectrin does not form a static network during myoblast fusion; instead, it forms a transient and dynamic structure that rapidly changes its density, shape and morphology in response to changes in the invasiveness of the PLS. The dynamic behavior of $\beta_{\mathrm{H}^{-}}$spectrin at the fusogenic synapse was confirmed by fluorescent recovery after bleaching (FRAP). When fluorescently labeled $\beta_{\mathrm{H}^{-}}$spectrin was photobleached at the fusogenic synapse as its accumulation reached equilibrium, the fluorescence rapidly recovered with an average $T_{1 / 2}$ of $59 \pm 18 \sec (n=6)$ and eventually reached $54 \pm 9 \%(n=6)$ of the pre-bleaching level (Fig. 2a, b; Supplementary Video 3). These results suggest that more than half of $\beta_{\mathrm{H}^{-}}$spectrin at the fusogenic synapse is in a mobile fraction and that $\beta_{\mathrm{H}}$-spectrin dynamically associates and dissociates at the fusogenic synapse, rather than being maintained in a stagnant structure. 
Given the correlation between spectrin accumulation and PLS invasiveness, we tested whether $\beta_{\mathrm{H}^{-}}$spectrin accumulation at the fusogenic synapse is triggered by mechanical force generated by the invasive PLS or is recruited by the cell adhesion molecules in the founder cell, Duf and its functionally redundant paralog Roughest $(\mathrm{Rst})^{21,22}$. We expressed a truncated form of Duf lacking its entire intracellular domain (Duf $\Delta$ intra) (Fig. 2c) in duf, rst double null mutant embryos and asked if $\beta_{\mathrm{H}^{-}}$-spectrin could still accumulate. Remarkably, $\beta_{\mathrm{H}}$-spectrin exhibited significant accumulation at the fusogenic synapse marked by the invasive F-actin foci and Duf $\Delta$ intra enrichment, albeit with a slight decrease in intensity compared with the wild-type control (Fig. 2d, f, g). In contrast, Antisocial (Ants)/Rols7, a founder cell adaptor protein that binds to the intracellular domain of Duf ${ }^{23-26}$, did not accumulate at the fusogenic synapse (Fig. 2, compare e and f; Fig. $2 \mathrm{~g}$ ). Thus, $\beta_{\mathrm{H}}$-spectrin accumulation in the founder cell can be triggered by invasive force from the PLS, independent of the chemical signaling from cell adhesion molecules. Consistent with these results, when cultured Drosophila S2R+ cells were induced to fuse by expressing the FCM-specific adhesion molecule Sticks and stones (Sns) and the C. elegans fusogen Eff- $1^{5}, \beta_{\mathrm{H}}$-spectrin also accumulated to the fusogenic synapse in response to the invasive protrusions, despite the lack of endogenous Duf and Rst in these cells ${ }^{5}$ (Fig. 2h).

To test directly whether $\beta_{\mathrm{H}^{-}}$spectrin exhibits mechanosensitive accumulation, we performed micropipette aspiration (MPA) assays, in which a pulling force is applied to the Drosophila S2 cell cortex by a micropipette with a diameter of $\sim 5 \mu \mathrm{m}$. Previously, we have shown that myosin II (Myoll) is a mechanosensory protein that accumulates to the tip of an aspirated S2 cell where the mechanical stress is high ${ }^{6}$. Interestingly, GFP- $\beta_{\mathrm{H}^{-}}$-spectrin also showed mechanosensitive accumulation, although unlike Myoll, GFP- $\beta_{\mathrm{H}}$-spectrin accumulated to the base area of the aspirated portion of the cell (Fig. 3a, d). This effect was not due to an increase in membranous 
materials in this area, since an RFP-tagged $\mathrm{PH}$ domain ${ }^{27}$, which interacts with phospholipids on the plasma membrane, did not accumulate at the base (Fig. 3b, d). Nor did this effect depend on the adaptor protein Ants that did not show mechanosensitive accumulation (Fig. 3b, d). In addition, no accumulation was observed for GFP- $\beta_{\mathrm{H}^{-}}$spectrin- $\Delta \mathrm{C}$ (Extended Data Fig. 3a), which deleted a C-terminal fragment containing the tetramerization domain ${ }^{28}$, or GFP- $\beta_{\mathrm{H}^{-}}$spectrin- $\Delta \mathrm{N}$ (Extended Data Fig. 3a), which deleted an N-terminal fragment containing the actin-binding domain $^{28}$ (Fig. 3b, d; Extended Data Fig. 3b), suggesting that the mechanosensitive accumulation of $\beta_{\mathrm{H}^{-}}$spectrin requires its tetramerization and actin-binding activities. Notably, $\beta_{\mathrm{H}^{-}}$ spectrin enrichment at the base is time- and force-dependent, which increased linearly over time until reaching its peak level at $80-90 \mathrm{sec}$ after the onset of aspiration (Extended Data Fig. 4a) and increased proportionally to applied pressure (Extended Data Fig. 4b). These results suggest that $\alpha / \beta_{H}$ spectrin binding to the actin network depends on the number of binding sites at a given time rather than additional cooperative activity of the previously bound tetramers, and that the mechanical force applied to the cortical actin network leads to an increase in the binding sites for the $\alpha / \beta_{H}$ spectrin heterotetramers.

It is intriguing that $\alpha / \beta_{\mathrm{H}^{-}}$spectrin and Myoll show distinct domains of mechanosensitive accumulation revealed by MPA. Previous coarse-grained modeling suggested that the tip of an aspirated cell corresponds to an area of maximal actin network dilation (or radial expansion), whereas the base area is under maximal shear deformation (or shape change) ${ }^{29}$. These analyses suggest that Myoll is a mechanosensory protein for actin network dilation, whereas $\alpha / \beta_{\mathrm{H}^{-}}$spectrin responds specifically to shear deformation. Consistent with the distinct areas of mechanosensitive accumulation of Myoll and spectrin, $\beta_{\mathrm{H}}$-spectrin remained accumulated to the base area in cells treated with Y27632, a small molecule that decreases Myoll activity by 
inhibiting Myoll's upstream activator ROCK (compare Fig. 3c, top and middle row; Fig. 3d), and Myoll remained accumulated at the tip of $\beta_{\mathrm{H}}$-spectrin knockdown cells (compare Fig. 3c, top and bottom row; Fig. 3e).

The distinct domains of mechanosensitive accumulation of Myoll and spectrin induced by pulling forces prompted us to ask whether they exhibit a similar response to pushing forces. Course-grained modeling of cells invaded by protrusions with a $5-\mu \mathrm{m}$ diameter predicted clear separation of dilation vs. shear domains along the invasive protrusion, with maximal dilation at the tip area and maximal shear deformation at the base (Fig. 3f). However, when the invasive protrusions became thinner (approaching a diameter of $400 \mathrm{~nm}$ ), there was a gradual increase of shear deformation at the tip area, where the dilation deformation remained largely the same (Fig. 3g-j). This model predicted that the mechanosensitive accumulations of spectrin and Myoll induced by thin protrusions may overlap at the tip area, although the maximal shear deformation still resided at the base. To test this directly, we performed atomic force microscopy (AFM) experiments, in which a pushing force was applied to cells expressing GFP- $\beta_{\mathrm{H}^{-}}$-spectrin and RFP-Myoll by a cantilever with a diameter of $\sim 200 \mathrm{~nm}$, closely mimicking the length scale of the invasion protrusions at the fusogenic synapse (Fig. 3k). $\beta_{\mathrm{H}}$-spectrin and Myoll exhibited rapid and largely overlapping domains of accumulation to the indented area by the cantilever (Fig. 3l; Supplementary Video 4), validating the pattern of mechanosensitive response predicted by the course-grained model. Consistent with this, both $\beta_{\mathrm{H}^{-}}$-spectrin and Myoll accumulated in largely overlapping domains together with Duf at the fusogenic synapse in founder cells of Drosophila embryos (Fig. 1k-m) ${ }^{6}$. 
What is the biological function of spectrin accumulation at the fusogenic synapse? In $\alpha / \beta_{H^{-}}$ spectrin double mutant embryos, the founder cell-specific adhesion molecule Duf and its interacting protein Ants were both dispersed along the muscle cell contact zone between two fusion partners, instead of forming a tight cluster at the fusogenic synapse as in wild-type embryos (Fig. 4a, b; Extended Data Fig. 5a, b). Time-lapse imaging revealed dynamically dispersing Duf at the fusogenic synapse in the $\alpha / \beta_{H^{-}}$spectrin mutant embryos (Supplementary Video 5), compared to the tight Duf cluster associated with dense F-actin foci in wild-type embryos (Fig. 4c; Supplementary Video 6). Occasionally, aggregated Duf clusters were initially observed in these mutant embryos, but in these cases Duf gradually diffused over time, suggesting that spectrin is required for the maintenance, but not the initiation, of the Duf cluster (Fig. 4d; Supplementary Video 5).

Since the cell adhesion molecules from the two fusion partners, Duf and Sns, interact in trans at the fusogenic synapse ${ }^{30}$, we tested whether Duf dispersal in founder cells of $\alpha / \beta_{H}$-spectrin mutant embryos affects Sns distribution in the FCM in a cell non-autonomous manner. Indeed, Sns was also dispersed along the muscle cell contact zone at the fusogenic synapse (Extended Data Fig. 5, compare c and d). Moreover, the actin nucleation promoting factors and their interacting proteins, such as the WASP-interacting protein (known as Solitary or Sltr), which are recruited by Sns to the fusogenic synapse ${ }^{31}$, were dispersed in these mutant embryos (Extended Data Fig. 5, compare e and f). Consequently, the F-actin structure generated by the FCM appeared diffused (Fig. 4b; Extended Data Fig. 5b, d, f), with an average fluorescent intensity of $61 \pm 19$ per focus on a $0-255$ scale $(n=35)$, compared to $170 \pm 15$ per focus $(n=28)$ in wild-type embryos. Electron microscopy studies revealed stubby and closely abutting toe-like

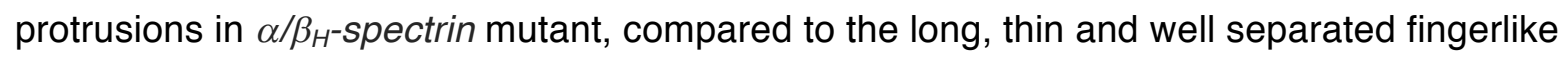


protrusions in wild-type embryos (Fig. $4 \mathrm{e}, \mathrm{f})^{4,17,18}$. Thus, Duf restriction by $\alpha / \beta_{\mathrm{H}^{-}}$spectrin in founder cells non-autonomously regulates Sns localization and the distribution of polymerized actin filaments along the cell contact zone at the fusogenic synapse in FCMs.

To investigate how spectrin maintains the Duf concentration at the fusogenic synapse, we performed co-immunoprecipitation experiments using Drosophila embryos expressing Flag- $\beta_{\mathrm{H}^{-}}$ spectrin and Duf-GFP specifically in the mesoderm. Antibody against Flag, but not a control antibody, co-precipitated both Duf-GFP and $\alpha$-spectrin, suggesting that $\alpha / \beta_{H^{-}}$spectrin restricts Duf via biochemical interactions (Fig. $4 \mathrm{~g}$ ). In the absence of spectrin-Duf interaction, such as in duf,rst mutant embryos expressing Duf $\Delta$ intra, even though the F-actin foci formed initially due to the trans-interaction between Duf $\Delta$ intra and Sns, the condensed foci eventually dispersed at the fusogenic synapse (Supplementary Video 7), similar to the phenotype of the $\alpha / \beta_{H^{-}}$spectrin mutant embryos (Fig. 4a, b; Extended Data Fig. 5b, d, f; Supplementary Video 5). Thus, the intracellular domain of Duf is required for stabilizing the mechanosensitive accumulation of spectrin, likely through spectrin-Duf interactions.

Besides restricting cell adhesion molecules, the abnormal morphology of the invasive protrusions in $\alpha / \beta_{H^{-}}$spectrin mutant embryos suggests that spectrin may play an additional role in shaping these intercellular protrusions. Using structured illumination (SIM) microscopy, we observed that the spectrin accumulation triggered by initial invasive protrusions did not form an evenly spaced network like in red blood cells and neuronal axons ${ }^{8-10}$. Instead, the network contained spectrin-enriched patches interspersed with spectrin-free domains (Fig. 4h-k). Interestingly, actin-propelled membrane protrusions only penetrated through the spectrin-free domains but not spectrin-enriched patches (Fig. 4h-k; Supplementary Video 8). The long and 
thin protrusions triggered additional mechanosensitive accumulation of spectrin at the tip and along the side (Fig. 4h, i), which may constrict further protrusions. These results suggest that the spectrin network in the founder cell functions as a "cellular sieve" to restrict the diameters of the invasive protrusions from the FCM. The resulting long and thin protrusions put the fusogenic synapse under high local tension and promote plasma membrane fusion ${ }^{4,6}$.

Taken together, this study has revealed a dynamic mechanosensitive response of spectrin and its cell non-autonomous function in shaping the architecture of invasive protrusions. During cellcell fusion, the mechanosensitive accumulation of spectrin in the receiving fusion partner sets up a transient spectrin-enriched network at the fusogenic synapse, which, in turn, restricts cell adhesion molecules via biochemical interactions and constricts the invasive protrusions to increase local tension and drive the fusion process (Extended Data Fig. 6). What triggers the mechanosensitive accumulation of spectrin? Previous studies using in vitro reconstituted actin networks have demonstrated that mechanical loading/compression increases the density of the actin network $^{32}$. We suggest that the invasive protrusions at the fusogenic synapse compress and deform the actin network in the receiving fusion partner, thus generating numerous additional spectrin binding sites and leading to its mechanosensitive accumulation. The $\alpha / \beta_{\mathrm{H}^{-}}$ spectrin bound to the dynamic actin network, in turn, may transiently stabilize the network by unfolding the spectrin repeats, as demonstrated in red blood cells under shear stress ${ }^{33}$. What makes spectrin dynamic at the fusogenic synapse? In many previously described cellular contexts where spectrin forms a static membrane skeletal structure, the spectrin-actin interaction is stabilized by accessory proteins, such as adducin ${ }^{34}$ and protein $4.1^{1,2,35,36}$. Interestingly, adducin and protein 4.1 were both absent at the fusogenic synapse (Extended Data Fig. 1b, c), which may account for the dynamic behavior of $\alpha / \beta_{\mathrm{H}^{-}}$spectrin during the fusion 
process. It is worth noting that the fusion-promoting function of spectrin is not fly-specific; it is conserved in mouse myoblast fusion (Extended Data Fig. 7). Given the widespread expression of spectrin in most eukaryotic cell types, our characterization of spectrin as a dynamic mechanosensory protein in fusogenic cells has broader implications for understanding spectrin functions in many dynamic cellular processes beyond cell-cell fusion. 

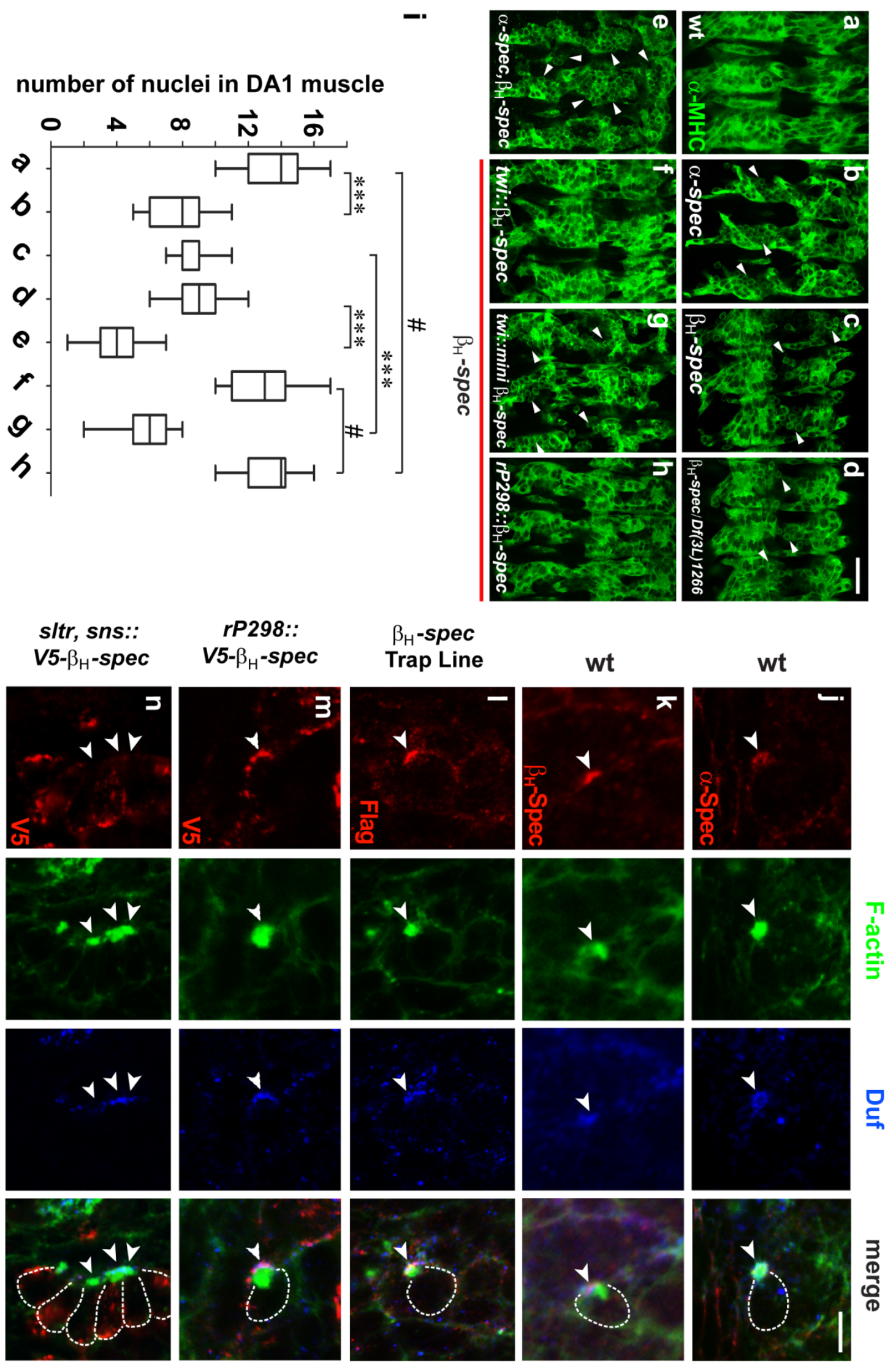


\section{Figure 1. Spectrin is required for myoblast fusion and enriched at the fusogenic synapse} in the receiving cell

(a-h) Myoblast fusion phenotype in $\alpha / \beta_{H^{-}}$spectrin mutant embryos. Somatic muscle cells in stage 15 embryos were labeled with anti-muscle myosin heavy chain (MHC) antibody. Ventral lateral muscles of three hemisegments are shown in each panel. Anterior is to the left and posterior to the right. (a) Wild-type (wt). (b-d) Minor fusion defect in $\alpha$-spectrin ( $\alpha$-spec) (b), $\beta_{H^{-}}$spectrin $\left(\beta_{H^{-}}\right.$ spec) (c), and transheterozygous $\beta_{H^{-}}$spec/Df(3L)1266 (d) mutant embryos. (e) Severe fusion defect in $\alpha / \beta_{H^{-}}$spectrin double mutant embryo. (f-h) Effect of $\beta_{H^{-}}$spec transgenes expression in $\beta_{H^{-}}$spec mutant embryos. Expression of full-length $\beta_{H^{-}}$spec in all muscle cells with a twi-GAL4 driver (f) or in founder cells with an rP298-GAL4 driver (h) rescued the fusion defect. Expression of mini- $\beta_{H^{-}}$spec, a dominant negative form of $\beta_{\mathrm{H}^{-}} \mathrm{Spec}$, in all muscle cells enhanced the fusion defect (g). Unfused cells are indicted by arrowheads. Scale bar, $20 \mu \mathrm{m}$.

(i) Quantification of the fusion index. The number of nuclei in the dorsal acute muscle 1 (DA1) were counted for each genotype in (a-h). Error bars indicate standard deviation. Statistical significance was determined by the Student's t test. ${ }^{* * *}, \mathrm{p}<0.001$; \#, not significant. (j-n) Localization of $\alpha / \beta_{\mathrm{H}^{-}}$spectrin at the fusogenic synapse. Stage 13-14 embryos were triple labeled with phalloidin (F-actin; green), anti-Duf (founder cell adhesion molecule; blue), as well as anti- $\alpha-\operatorname{Spec}(\mathrm{j})$, anti- $\beta_{\mathrm{H}}-\mathrm{Spec}(\mathrm{k})$, anti-Flag $\left(\beta_{\mathrm{H}}\right.$-spec-Flag trap; I), anti-V5 (V5- $\beta_{\mathrm{H}}$-Spec expressed in founder cell; $\mathrm{m})$ and anti-V5 (V5- $\beta_{\mathrm{H}^{-}}$Spec expressed in FCM with an sns-GAL4 driver; $n$ ). Note the accumulation of $\alpha-S p e c(j)$ and $\beta_{H}-S p e c$ (k and I) at the fusogenic synapse and their colocalization with Duf. The white outlines in the merged panels delineate FCMs which attacked founder cells at the fusogenic synapse. Scale bar, $5 \mu \mathrm{m} .(m-n)$ Founder cell-specific accumulation of $\beta_{\mathrm{H}-S p e c}$ at the fusogenic synapse. V5-tagged $\beta_{\mathrm{H}}$-Spec was specifically expressed in founder cells $(m)$ or in FCMs $(n)$ and visualized by anti-V 5 staining. Expression of 
$\beta_{\mathrm{H}-S p e c}$ in FCMs was performed in a fusion-defective sltr mutant to prevent diffusion of ectopically expressed $\beta_{\mathrm{H}}$-Spec proteins from FCMs to founder cells following myoblast fusion events. Note that $\beta_{\mathrm{H}}-\mathrm{Spec}$ was accumulated in founder cells $(m)$, not in FCMs $(n)$, at the fusogenic synapse. 

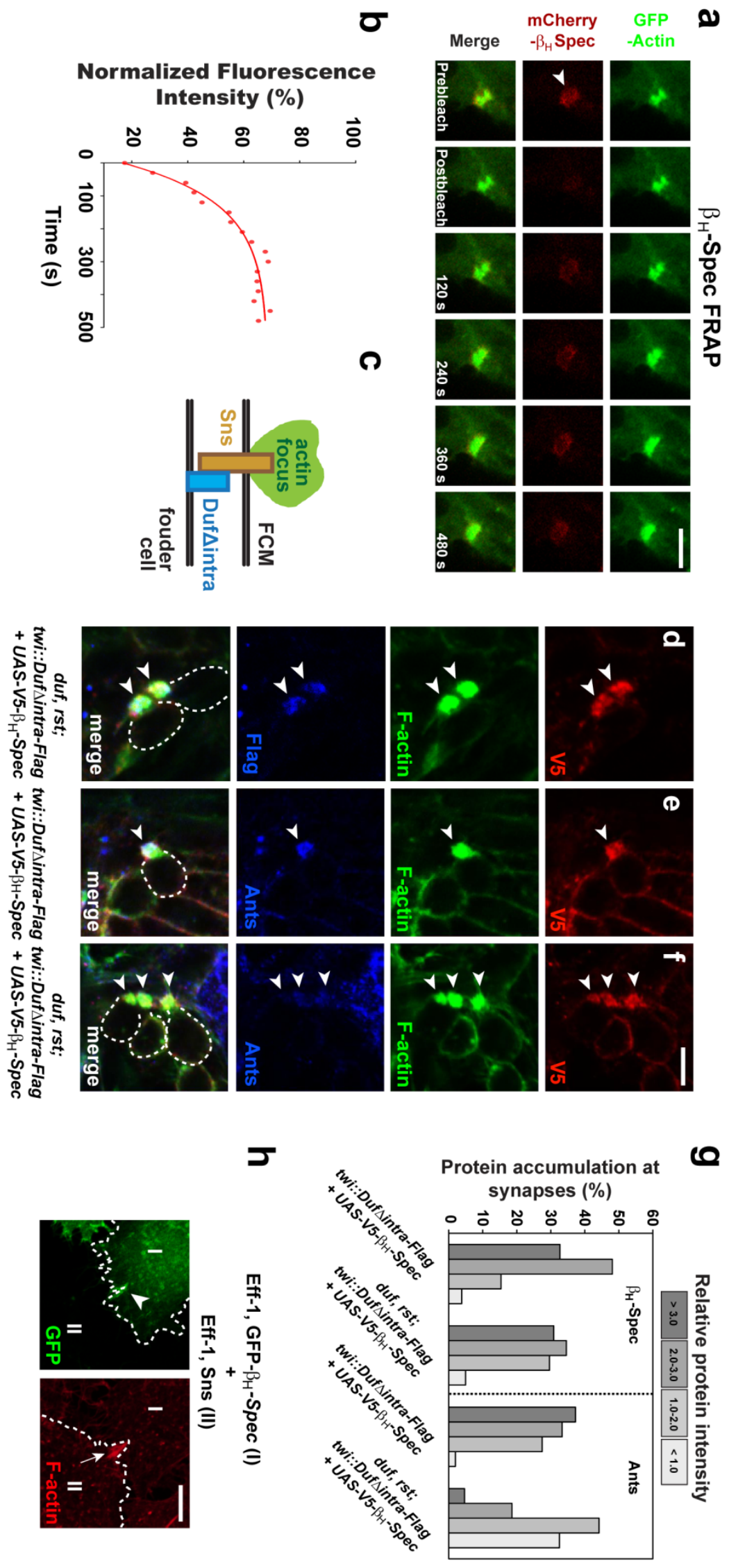
Figure 2. Spectrin dynamically accumulates at the fusogenic synapse in the absence of chemical signaling from cell adhesion molecules

(a-b) FRAP of $\beta_{\mathrm{H}^{-}}$Spec at the fusogenic synapse. (a) Stills from time-lapse imaging of a stage 14 wt embryo expressing GFP-actin and mCherry- $\beta_{\mathrm{H}}-\mathrm{Spec}$. Arrowhead indicates the photobleached region. Scale bar, $5 \mu \mathrm{m}$. (b) Recovery kinetics of the mCherry fluorescence after photobleaching.

(c-g) $\beta_{\mathrm{H}}-$ Spec accumulation at the fusogenic synapse in the absence of chemical signaling. (c) $\mathrm{A}$ schematic drawing of the fusogenic synapse showing truncated Duf (Duf $\Delta$ intra) in the founder cell interacting with Sns in FCM to induce the formation of an invasive F-actin focus. (d-f) Coexpression of $\mathrm{V} 5-\beta_{\mathrm{H}}-\mathrm{Spec}$ (red) and Duf $\Delta$ intra-Flag (blue) in all muscle cells of duf,rst double mutant ( $d$ and $f$ ) and wt (e) embryos. The FCMs are outlined in the merge panels. Note the $\beta_{\mathrm{H}^{-}}$ Spec accumulation ( $d$ and $f$ ) and the lack of accumulation of Ants (anti-Ants; blue; $f$ ) at the fusogenic synapse (arrowheads) in the absence of Duf intracellular signaling. (g) Quantification of the relative intensity of $\beta_{\mathrm{H}}-\mathrm{Spec}$ and Ants enrichment at the fusogenic synapse in wt and Duf $\Delta$ intra-expressing duf, rst double mutant embryos. The fluorescent intensity at the fusogenic synapse was compared with that in the adjacent cortical area to calculate the relative protein intensity ratio ( $n>40$ for each genotype). Scale bar, $5 \mu \mathrm{m}$.

(h) $\beta_{\mathrm{H}^{-}}$Spec enrichment at the fusogenic synapse in fusing S2R+ cells. GFP- $\beta_{\mathrm{H}^{-}}$Spec expressed in a receiving cell (I; outlined) accumulated at the fusogenic synapse (arrowhead) in response to an F-actin-propelled invasive protrusion (arrow) from an attacking cell (II; outlined). Scale bar, 5 $\mu \mathrm{m}$. 
a

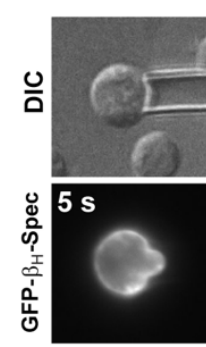

b

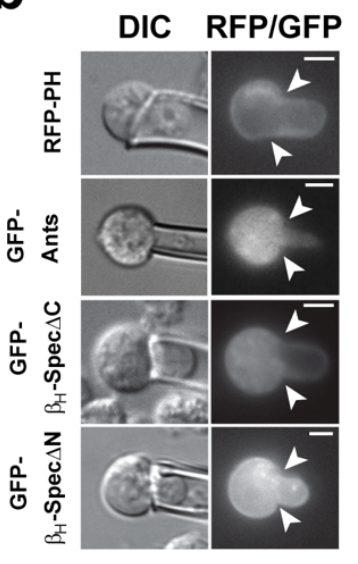

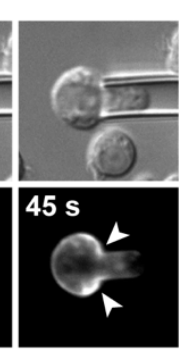
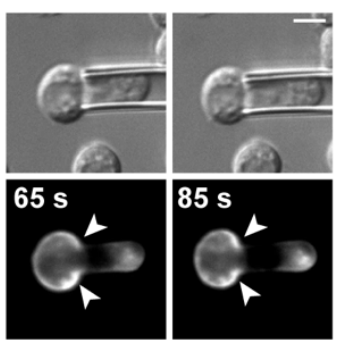

C
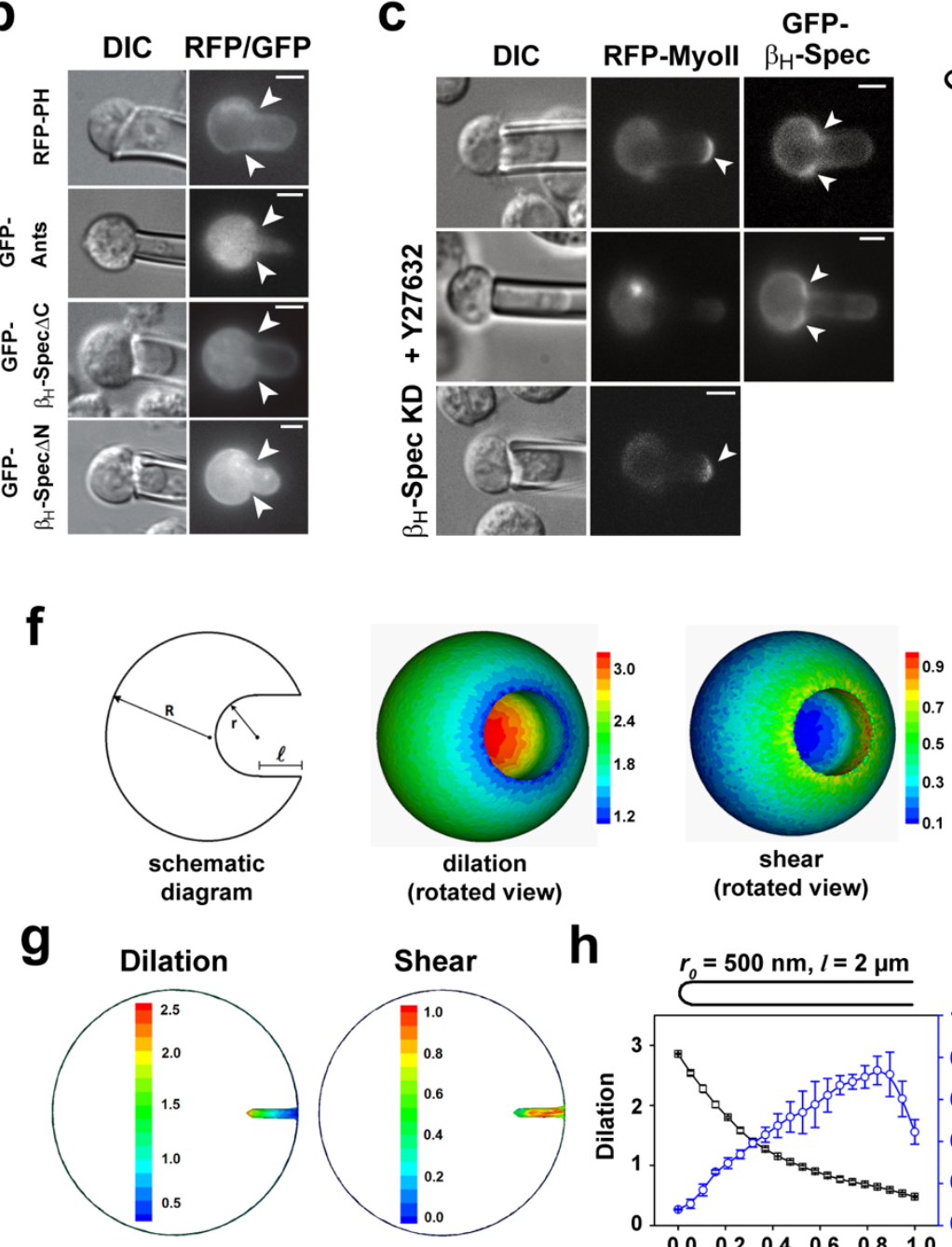

h
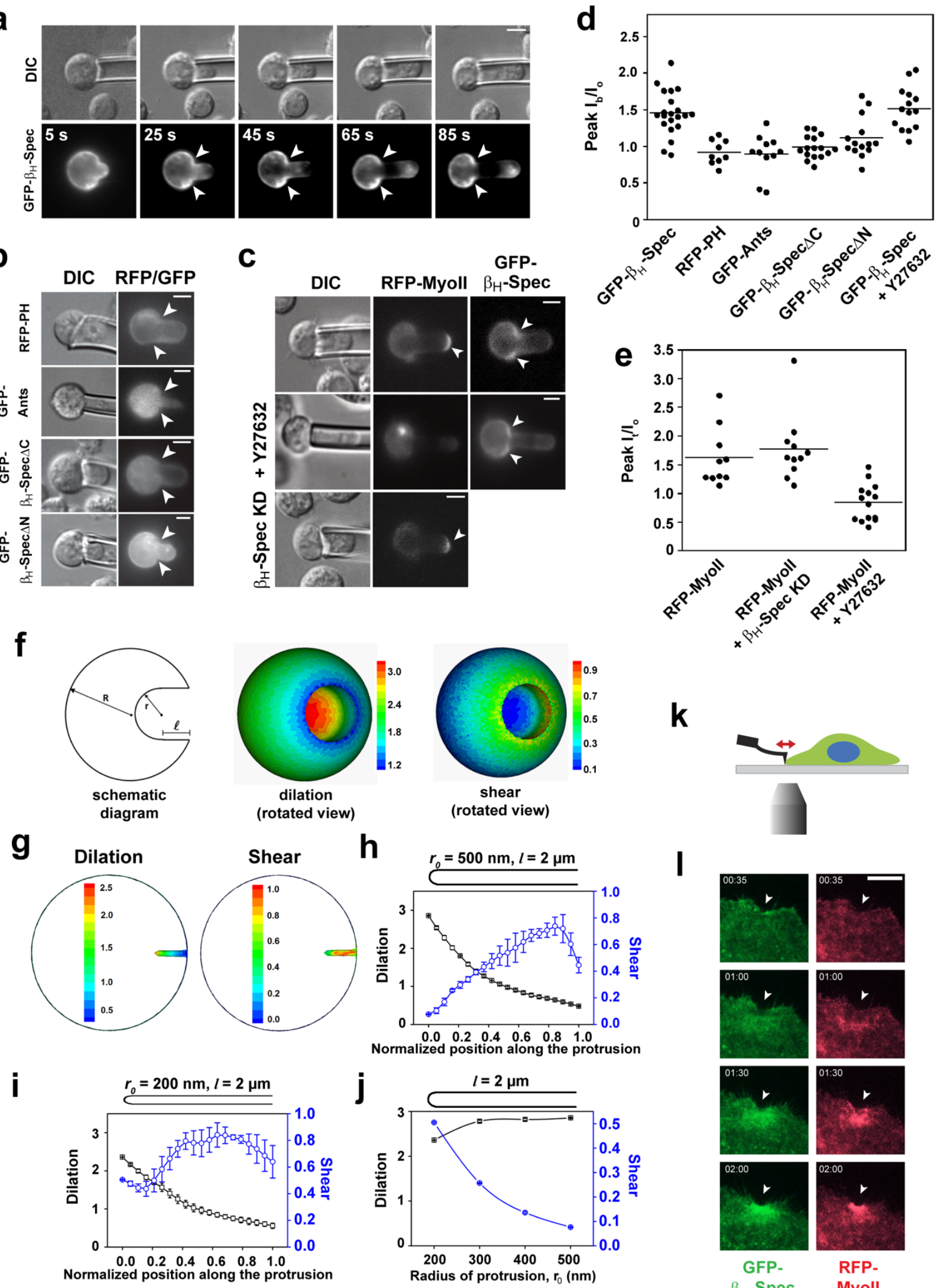

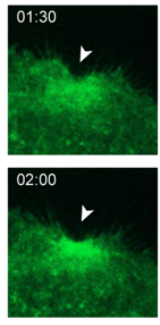

GFP$\beta_{\mathrm{H}}$-Spec

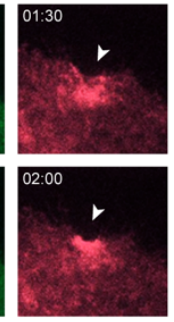

RFPMyoll 
Figure 3. Spectrin exhibits mechanosensitive accumulation to areas of shear deformation (a-e) Mechanosensitive accumulation of $\beta_{\mathrm{H}}$-Spec revealed by MPA. (a) Stills of time-lapse imaging of an aspirated S2 cell expressing GFP- $\beta_{\mathrm{H}^{-}}$Spec. Differential interference contrast (DIC) and fluorescent images are shown in the top and bottom panels, respectively. Note the enrichment of $\beta_{\mathrm{H}^{-}}$Spec to the base area (arrowheads) of the aspirated portion of the cell. (b) No mechanosensitive accumulation of a RFP-PH domain (membrane marker), GFP-Ants, GFP- $\beta_{\mathrm{H}^{-}}$ Spec $\Delta$ C and GFP- $\beta_{H^{-}}$Spec $\Delta N$. (c) Spatially distinct and independent mechanosensory accumulation of $\beta_{\mathrm{H}^{-}}$Spec and Myoll. In the control cell (top row), Myoll was enriched at the tip of the aspirated portion of the cell (arrowhead, top row, middle panel) and $\beta_{\mathrm{H}}$-Spec enriched at the base (arrowheads, top row right panel). Suppressing Myoll activity by Y27632 abolished the accumulation of Myoll (middle row, middle panel), but not $\beta_{\mathrm{H}^{-}}$Spec (arrowheads, middle row, right panel), whereas $\beta_{\mathrm{H}^{-}}$Spec knockdown (KD) did not affect Myoll accumulation (bottom row). Scale bars, $5 \mu \mathrm{m}$. (d) Quantification of protein accumulation at the base of aspirated cells. Background-subtracted fluorescent intensities at the base $\left(I_{b}\right)$ and at the opposite pole of the cell body $\left(\mathrm{I}_{0}\right)$ were measured, and the ratio $\left(\mathrm{I}_{\mathrm{b}} / \mathrm{I}_{0}\right)$ was calculated. (e) Quantification of Myoll accumulation at the tip of aspirated cells. Background-subtracted fluorescent intensities at the tip $\left(\mathrm{I}_{\mathrm{t}}\right)$ and at the opposite pole of the cell body $\left(\mathrm{I}_{\mathrm{o}}\right)$ were measured, and the ratio $\left(\mathrm{I}_{\mathrm{t}} / \mathrm{I}_{\mathrm{o}}\right)$ was calculated. In (d) and (e), each data point represents a cell and each horizontal line indicates the average ratio.

(f-i) Coarse-grained simulation of deformation in the receiving cell induced by invasive protrusions.

(f, left panel) Schematic graph of the geometry of a protrusion on the surface of a receiving cell. ( $f$ and $g$ ) Heat maps of the simulated dilation ( $f$, middle panel and $g$, left panel) and shear ( $f$, and 
$\mathrm{g}$, right panels) deformations induced by protrusions with a radius $\left(r_{0}\right)$ of $2.5 \mu \mathrm{m}$ (F' and F") and $200 \mathrm{~nm}$ (G and G'), respectively.

(h and i) Plots of dilation and shear deformation along protrusions with an $r_{0}$ of $500 \mathrm{~nm}(\mathrm{H})$ and $200 \mathrm{~nm}(\mathrm{I})$. Each data point is the average deformation of 4 different lines along the protrusions. Note that the shear deformation at the tip region increases in (i) compared to (h).

(j) Dilation and shear deformations at the tip area (0.0-0.2) of protrusions with different $r_{0}$. Note the gradual increase in shear deformation at the tip with a decrease in the protrusion radius. The length (I) of all protrusions is $2 \mu \mathrm{m}$.

( $\mathrm{k}$ and $\mathrm{I}) \beta_{\mathrm{H}^{-}}$Spec accumulation in response to mechanical stress revealed by AFM. $(\mathrm{k})$ Schematic drawing of the AFM experiments. A cantilever applied a pushing force to the cortex of an S2R+ cell. (I) Stills from live imaging of an S2R+ cell expressing GFP- $\beta_{\mathrm{H}}-\mathrm{Spec}$ and RFPMyoll. Both $\beta_{\mathrm{H}^{-}}$Spec and Myoll rapidly accumulated to the indented area generated by the cantilever (arrowheads). Scale bar, $10 \mu \mathrm{m}$. 

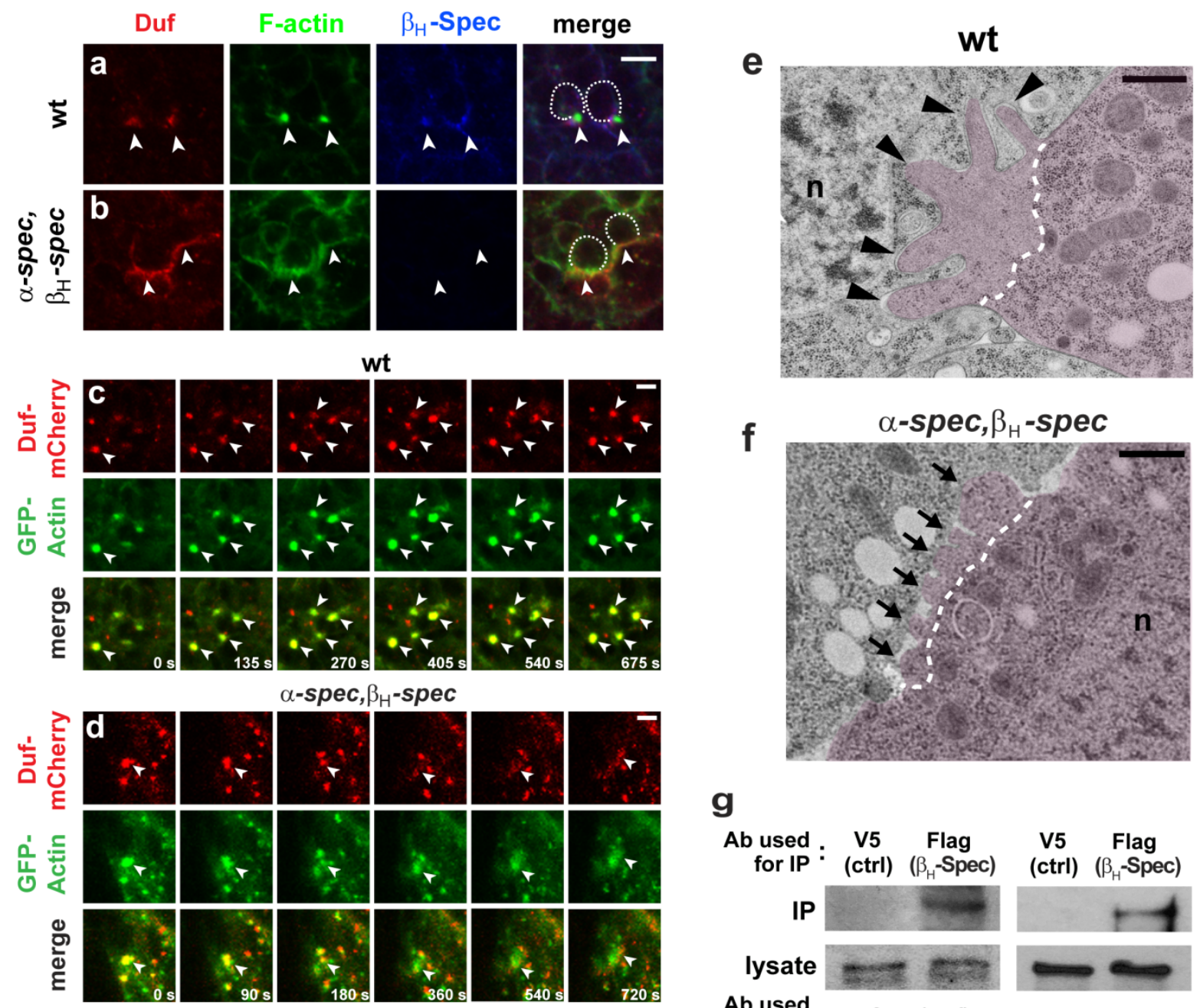

g

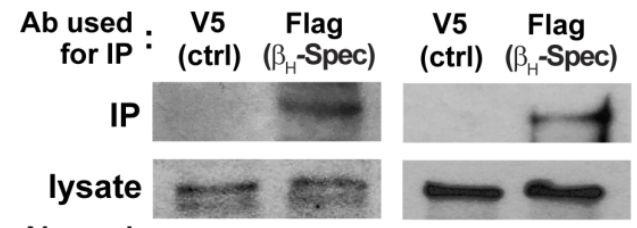

Ab used :
for IB $\quad$ GFP (Duf) $\quad \alpha-S p e c$

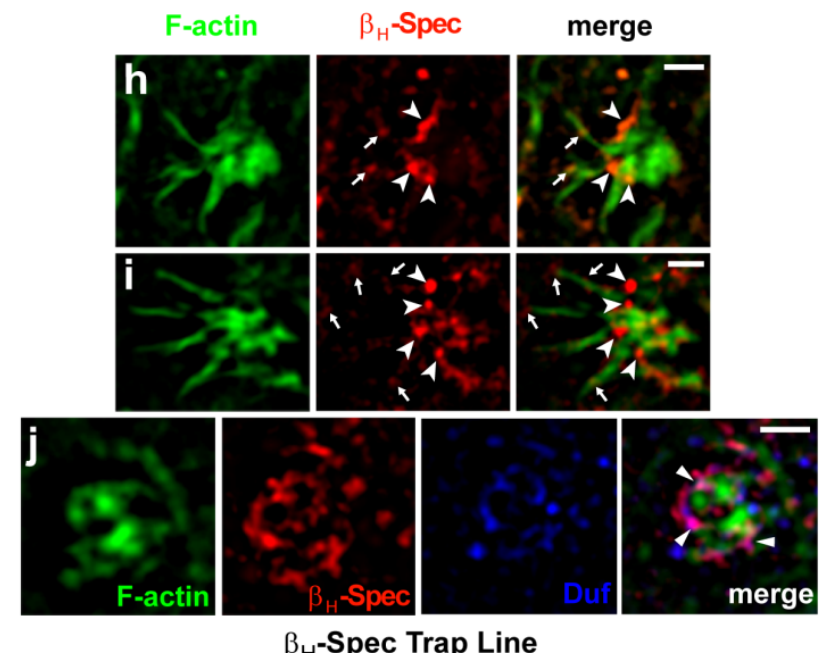

$\mathbf{k}$

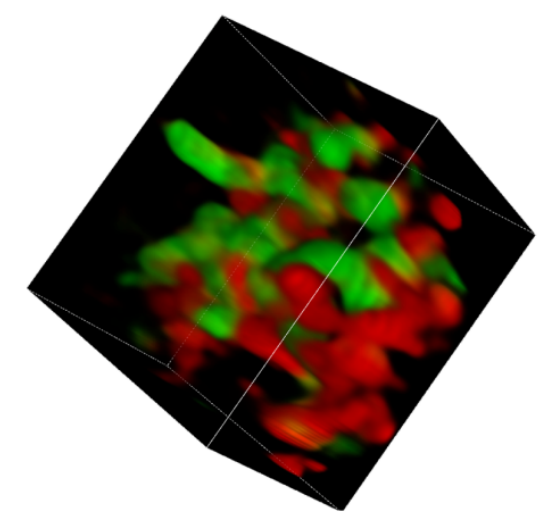

Figure 4. The spectrin network restricts cell adhesion molecules and constricts invasive protrusions at the fusogenic synapse 
(a-b) Duf is dispersed at the fusogenic synapse in $\alpha / \beta_{H}$-spectrin double mutant embryos. Stage 14 embryos labeled with anti-Duf (red), phalloidin (F-actin) and anti- $\beta_{\mathrm{H}^{-}}$Spec (blue). (a) Duf is concentrated in a tight cluster at the fusogenic synapse (arrowheads) with the dense F-actin focus in a wt embryo. (b) Duf is dispersed along the muscle cell contact zone (arrowheads) with fuzzy actin filaments in a $\alpha / \beta_{H^{-}}$spectrin mutant embryo. FCMs are outlined in the merge panels. Scale bar, $5 \mu \mathrm{m}$.

(c-d) Stills from time-lapse movies of wt (c) or $\alpha / \beta_{H^{-}}$spectrin mutant (d) embryos expressing DufmCherry (red) and GFP-actin (green). Arrowheads indicate fusogenic synapses. Note that Duf remained in a tight cluster associated with the dense F-actin foci in wt, but diffused over time accompanied by the dispersal of the F-actin focus in an $\alpha / \beta_{H^{-}}$-spectrin mutant embryo. Scale bar, $5 \mu \mathrm{m}$.

(e-f) Electron micrographs of the invasive PLS in wt (e) and $\alpha / \beta_{H^{-}}$spectrin mutant (f) embryos. FCMs were pseudo-colored in purple. Dash lines delineate the F-actin enriched area of the PLS. Note the long, thin and well separated fingerlike protrusions in wt (arrowheads in e) and the stubby and closely abutting toe-like protrusions in $\alpha / \beta_{H}$-spectrin mutant (arrows in f) embryos. $\mathrm{n}$, nucleus. Scale bars, $500 \mathrm{~nm}$.

(g) Biochemical interaction between $\beta_{\mathrm{H}}-\mathrm{Spec}$ and Duf. Co-immunoprecipitation (IP) experiment using extracts from embryos expressing Flag- $\beta_{H^{-}}$Spec and Duf-GFP. Duf was pulled down with anti-Flag antibody, suggesting that $\beta_{\mathrm{H}}$-Spec interacts with Duf. $\alpha$-Spec was detected to indicate the presence of $\beta_{\mathrm{H}^{-}}$Spec, the latter of which was difficult to detect due to its large size ( 480 $\mathrm{kDa})$.

(h-j) The spectrin network constricts the actin-propelled protrusions. Structural illuminated microscopy (SIM) images of fusogenic synapses, labeled with F-actin (green) and $\beta_{\mathrm{H}^{-}}$Spec (red). (h and i) Side-by-side view of two distinct fusogenic synapses between an FCM (right) 
and a founder cell (left). Note that the long and thin actin-propelled protrusions from the FCM penetrated through spectrin-free domains, but not spectrin-enriched patches (arrowheads), within the network. The long and thin protrusions triggered additional accumulation of spectrin at the tips or along the protrusions (arrows). (j) Top-to-bottom view of a fusogenic synapse. Note that the F-actin enriched areas were non-overlapping with those of $\beta_{\mathrm{H}}$-Spec or Duf (blue). Also note the significant overlapping domains of $\beta_{\mathrm{H}}$ Spec and Duf (arrowheads).

(k) 3D reconstruction of the fusogenic synapse, highlighting the mutually exclusive localization of F-actin and $\beta_{\mathrm{H}^{-}}$Spec. 


\section{REFERENCES}

1 Bennett, V. \& Lorenzo, D. N. Spectrin- and ankyrin-based membrane domains and the evolution of vertebrates. Curr Top Membr 72, 1-37 (2013).

2 Bennett, V. \& Lorenzo, D. N. An Adaptable Spectrin/Ankyrin-Based Mechanism for Long-Range Organization of Plasma Membranes in Vertebrate Tissues. Curr Top Membr 77, 143-184 (2016).

3 Machnicka, B. et al. Spectrins: a structural platform for stabilization and activation of membrane channels, receptors and transporters. Biochim Biophys Acta 1838, 620-634 (2014).

4 Sens, K. L. et al. An invasive podosome-like structure promotes fusion pore formation during myoblast fusion. J Cell Biol 191, 1013-1027 (2010).

5 Shilagardi, K. et al. Actin-propelled invasive membrane protrusions promote fusogenic protein engagement during cell-cell fusion. Science 340, 359-363 (2013).

$6 \mathrm{Kim}, \mathrm{J}$. H. et al. Mechanical tension drives cell membrane fusion. Dev Cell 32, 561-573 (2015).

7 Kim, J. H., Jin, P., Duan, R. \& Chen, E. H. Mechanisms of myoblast fusion during muscle development. Curr Opin Genet Dev 32, 162-170 (2015).

8 Liu, S. C., Derick, L. H. \& Palek, J. Visualization of the hexagonal lattice in the erythrocyte membrane skeleton. J Cell Biol 104, 527-536 (1987).

9 Pielage, J. et al. A presynaptic giant ankyrin stabilizes the NMJ through regulation of presynaptic microtubules and transsynaptic cell adhesion. Neuron 58, 195-209 (2008).

10 Byers, T. J. \& Branton, D. Visualization of the protein associations in the erythrocyte membrane skeleton. Proc Natl Acad Sci U S A 82, 6153-6157 (1985).

11 Xu, K., Zhong, G. \& Zhuang, X. Actin, spectrin, and associated proteins form a periodic cytoskeletal structure in axons. Science 339, 452-456 (2013). 
12 Hammarlund, M., Jorgensen, E. M. \& Bastiani, M. J. Axons break in animals lacking beta-spectrin. J Cell Biol 176, 269-275 (2007).

13 Krieg, M., Dunn, A. R. \& Goodman, M. B. Mechanical control of the sense of touch by beta-spectrin. Nat Cell Biol 16, 224-233 (2014).

14 Chen, E. H. \& Olson, E. N. Unveiling the mechanisms of cell-cell fusion. Science $\mathbf{3 0 8}$, 369-373 (2005).

15 Aguilar, P. S. et al. Genetic basis of cell-cell fusion mechanisms. Trends Genet 29, 427437 (2013).

16 Shin, N. Y. et al. Dynamin and endocytosis are required for the fusion of osteoclasts and myoblasts. J Cell Biol 207, 73-89 (2014).

17 Jin, P. et al. Competition between Blown Fuse and WASP for WIP Binding Regulates the Dynamics of WASP-Dependent Actin Polymerization In Vivo. Dev Cell 20, 623-638 (2011).

18 Duan, R. et al. Group I PAKs function downstream of Rac to promote podosome invasion during myoblast fusion in vivo. J Cell Biol 199, 169-185 (2012).

19 Thomas, G. H. \& Kiehart, D. P. Beta heavy-spectrin has a restricted tissue and subcellular distribution during Drosophila embryogenesis. Development 120, 2039-2050 (1994).

20 Tjota, M. et al. Annexin B9 binds to beta $(\mathrm{H})$-spectrin and is required for multivesicular body function in Drosophila. J Cell Sci 124, 2914-2926 (2011).

21 Ruiz-Gomez, M., Coutts, N., Price, A., Taylor, M. V. \& Bate, M. Drosophila dumbfounded: a myoblast attractant essential for fusion. Cell 102, 189-198. (2000).

22 Strunkelnberg, M. et al. rst and its paralogue kirre act redundantly during embryonic muscle development in Drosophila. Development 128, 4229-39 (2001). 
23 Chen, E. H. \& Olson, E. N. Antisocial, an intracellular adaptor protein, is required for myoblast fusion in Drosophila. Dev Cell 1, 705-715 (2001).

24 Menon, S. D. \& Chia, W. Drosophila rolling pebbles: a multidomain protein required for myoblast fusion that recruits $\mathrm{D}$-Titin in response to the myoblast attractant Dumbfounded. Dev Cell 1, 691-703 (2001).

25 Rau, A. et al. rolling pebbles (rols) is required in Drosophila muscle precursors for recruitment of myoblasts for fusion.PG - 5061-73. Development 128 (2001).

26 Menon, S. D., Osman, Z., Chenchill, K. \& Chia, W. A positive feedback loop between Dumbfounded and Rolling pebbles leads to myotube enlargement in Drosophila. J Cell Biol 169, 909-920 (2005).

27 Stauffer, T. P., Ahn, S. \& Meyer, T. Receptor-induced transient reduction in plasma membrane PtdIns(4,5)P2 concentration monitored in living cells. Curr Biol 8, 343-346 (1998).

28 Bennett, V. \& Healy, J. Membrane domains based on ankyrin and spectrin associated with cell-cell interactions. Cold Spring Harb Perspect Biol 1, a003012 (2009).

29 Luo, T., Mohan, K., Iglesias, P. A. \& Robinson, D. N. Molecular mechanisms of cellular mechanosensing. Nat Mater 12, 1064-1071 (2013).

30 Galletta, B. J., Chakravarti, M., Banerjee, R. \& Abmayr, S. M. SNS: Adhesive properties, localization requirements and ectodomain dependence in S2 cells and embryonic myoblasts. Mech Dev 121, 1455-1468 (2004).

$31 \mathrm{Kim}, \mathrm{S}$. et al. A critical function for the actin cytoskeleton in targeted exocytosis of prefusion vesicles during myoblast fusion. Dev Cell 12, 571-586 (2007).

32 Bieling, P. et al. Force Feedback Controls Motor Activity and Mechanical Properties of Self-Assembling Branched Actin Networks. Cell 164, 115-127 (2016). 
33 Krieger, C. C. et al. Cysteine shotgun-mass spectrometry (CS-MS) reveals dynamic sequence of protein structure changes within mutant and stressed cells. Proc Natl Acad Sci U S A 108, 8269-8274 (2011).

34 Gardner, K. \& Bennett, V. Modulation of spectrin-actin assembly by erythrocyte adducin. Nature 328, 359-362 (1987).

35 Ungewickell, E., Bennett, P. M., Calvert, R., Ohanian, V. \& Gratzer, W. B. In vitro formation of a complex between cytoskeletal proteins of the human erythrocyte. Nature 280, 811-814 (1979).

36 Bennett, V. \& Baines, A. J. Spectrin and ankyrin-based pathways: metazoan inventions for integrating cells into tissues. Physiol Rev 81, 1353-1392 (2001). 


\section{ACKNOWLEDGEMENTS}

We thank the Bloomington stock center for fly stocks, B. Paterson for the MHC antibody, Guofeng Zhang for help with HPF/FS, J. Nathans for sharing confocal microscopes, and D. Pan for critically reading the manuscript. This work was supported by NIH grants (R01 AR053173 and R01 GM098816), American Heart Association Established Investigator Award and HHMI Faculty Scholar Award to E.H.C.; NIH grants (R01 GM66817 and R01 GM109863) to D.N.R; NIH grants (R01 GM074751 and R01 GM114671) to D.A.F; NSF grant \#MCB-1122013 to G.T.; and NSFC grant \#11572316 to T.L. R.D. was supported by an AHA postdoctoral fellowship; K.S. by an AHA Scientist Development Grant; S.S. by a Life Sciences Research Foundation postdoctoral fellowship; and D. L. by a Canadian Institute of Health Research postdoctoral fellowship.

\section{AUTHOR CONTRIBUTIONS}

R.D. initiated the project. R.D., J.K., K.S. and E.C. planned the project, performed experiments in Figures 1, 2, 4 (a-d, g) and Extended Data Figures 2, 3, 5, 7, and discussed the data. J.K. and E.C. collaborated with E.S. and D.R. on the MPA experiments and with S.S. and D.F. on the AFM experiments. D.L carried out the SIM experiments. S.L. carried out the EM experiments. T.L. contributed the coarse-grained models. G.T. contributed a spectrin construct. R.D., J.K., K.S. and E.C. made the figures. J.K and E.C. wrote the paper. All authors commented on the manuscript.

\section{AUTHOR INFORMATION}

The authors declare no competing financial interests. Correspondence and requests for materials should be addressed to E.H.C (Elizabeth.Chen@UTSouthwestern.edu). 


\section{METHODS}

\section{Fly genetics}

Fly stocks used in this study: $\alpha-\operatorname{spec}^{r g 41}$ ( $\alpha$-spec mutant; Bloomington Stock Center), $k s t^{14.1}\left(\beta_{H^{-}}\right.$ spec mutant $)^{19}$, Df(3L)1226 $\left(\beta_{H^{-}} \text {Spec deficiency line }\right)^{19}$, UAS-mini- $\beta_{H^{-}}$Spec ${ }^{37}$, kst $^{\text {MIO03134-GFSTF.1 }}\left(\beta_{H^{-}}\right.$

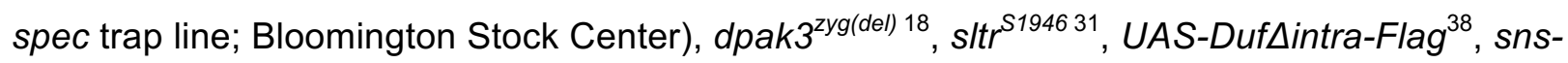
GAL4 ${ }^{39}, r P 298-G A L 4^{24}$, UAS-GFP-Actin and UAS-Actin-mRFP (Bloomington Stock Center). Transgenic flies carrying UAS-V5- $\beta_{H}-S p e c$, UAS-mCherry- $\beta_{H}-S p e c$, UAS-Duf-GFP and UASDuf-mCherry were generated by P-element-mediated germline transformation. To express genes in muscle cells, females carrying the transgene under the control of an UAS promoter were crossed with twi-GAL4 (all muscle cells), rP298-GAL4 (founder cells) and sns-GAL4 (FCMs) males, respectively. $\alpha / \beta_{H}$-spectrin double mutant, $\alpha$-spec ${ }^{r 441}, k s t^{14.1} / T M 6$ (labeled as $\alpha$ spec, $\beta_{H^{-}}$spec in figures), was generated using standard genetic methods.

\section{Molecular biology}

Full-length $\beta_{H}$-spec was amplified by PCR (with or without a tag) from cDNAs generated from mRNA of stage $11-15 w^{1118}$ flies. Due to the large size of the $\beta_{H}-s p e c$ gene, three fragments were individually amplified using the primers as follows:

(1) $\beta_{\mathrm{H}}$-spec-5': GACCGGTCAACATGACCCAGCGGGACGGCATC

(2) $\beta_{H}$-spec-3721-3': CTCCACGAATTCGGTGTCATG

(3) $\beta_{H^{-}}$spec-3721-5': CATGACACCGAATTCGTGGAG

(4) $\beta_{\mathrm{H}}$-spec-8214-3': CTCACCCTCTAGAATGCTATTG

(5) $\beta_{H}$-spec-8214-5': CAATAGCATTCTAGAGGGTGAG

(6) $\beta_{\mathrm{H}}$-spec-3': CCAAGCGGCCGCTCACTGTGGCGGGACTTGACTC 
The three PCR fragments were then subcloned into a Drosophila transformation vector pUAST.

To generate the UAS- $\beta_{H^{-}}$Spec $\Delta \mathrm{N}$ and UAS- $\beta_{H^{-}}$Spec $\Delta \mathrm{C}$ constructs, the following primers were used:

(1) $\beta_{H}$-spec-3865-5’: GGAATTCCAACATGGTGTGTCGATCTGCAAATGTTC

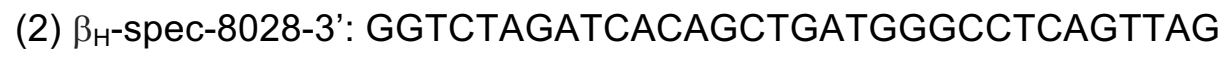

To generate pDEST- $\beta_{H}$ Spec constructs for GST-fusion proteins for the F-actin cosedimentation assays, the Gateway cloning system was used (Invitrogen)with the following primers:

(1) $\beta_{\mathrm{H}}-\mathrm{spec}-1-\mathrm{C}$ :

GGGGACAAGTTTGTACAAAAAAGCAGGCTTCATGACCCAGCGGGACGGCATC

(2) $\beta_{\mathrm{H}}$-spec-1-K:

GGGGACCACTTTGTACAAGAAAGCTGGGTTTTACTTCTTGCGATCTGCGTCCAT

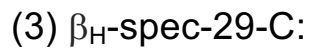

GGGGACAAGTTTGTACAAAAAAGCAGGCTTCGGAGCCAAACAAGTCCACGTC

(4) $\beta_{H^{-}}$spec-31-K:

GGGGACCACTTTGTACAAGAAAGCTGGGTTTTATTGGGACGCCGCATTCTGGCG

(5) $\beta_{H}$-spec-34-C:

GGGGACAAGTTTGTACAAAAAAGCAGGCTTCCCGAACATGCAACTGCTTAGC

(6) $\beta_{\mathrm{H}}$-spec-34-K:

GGGGACCAC TTTGTACAAGAAAGCTGGGTTTTATCACTGTGGCGGGACTTGACT dsRNAs were synthesized by in vitro transcription with gene-specific primers containing the T7 promoter sequence (TTAATACGACTCACTATAGGGAGA) at the 5' end (MEGAscript; Ambion). Synthesized dsRNAs were purified using NucAway ${ }^{\mathrm{TM}}$ Spin Columns (Ambion).

Immunofluorescent staining and imaging 
Fly embryos were fixed and stained as described previously ${ }^{4,31}$. The following primary antibodies were used: rabbit $\alpha$-muscle myosin heavy chain (1:1000) (gift from B. Paterson), rabbit anti- $\beta_{\mathrm{H}}$-Spectrin $(1: 100)^{19}$, rabbit anti- $\beta$-Spectrin $(1: 400)^{40}$, mouse anti- $\alpha$-Spectrin $(1: 1$; DSHB), guinea pig $\alpha$-Duf $(1: 500)^{4}$, guinea pig $\alpha$-Ants $(1: 1000)^{23}$, rat $\alpha$-SItr $(1: 30)^{31}$, rat $\alpha$-Sns $(1: 500)^{41}$, mouse anti-adducin (1:400; DSHB), mouse anti-protein 4.1 (1:400; DSHB), mouse $\alpha$ Flag (1:200; Sigma) and mouse $\alpha$-V5 (1:200; Invitrogen). The following secondary antibodies were used at 1:200: Alexa488- (Invitrogen), Cy3-, and Cy5- (Jackson Laboratories) conjugated and biotinylated (Vector Laboratories) antibodies made in goats. For phalloidin staining, FITCor Alexa568-conjugated phalloidin (Invitrogen) were used at 1:200. Fluorescent images were obtained on an LSM 700 Meta confocal microscope (Zeiss), acquired with LSM Image Browser software (Zeiss) and Zen software (Zeiss), and processed using Adobe Photoshop CS. For quantification of fluorescent signals, the signal intensities of cellular area of interest and control area were measured and processed for presentation by the Image $\mathrm{J}$ program (http://imagej.nih.gov/ij/).

\section{Drosophila cell culture}

S2 and S2R+ cells were cultured in Schneider's medium (Gibco) supplemented with 10\% fetal bovine serum (FBS) (Gibco) and penicillin/streptomycin (Sigma). Cells were transfected using Effectene (Qiagen) per the manufacturer's instructions. For RNAi knockdown, cells were first incubated with 3-5 $\mu \mathrm{g} / \mathrm{ml}$ of dsRNA for 2-4 days and then transfected with appropriate DNA constructs.

For immunofluorescent staining, cells were fixed with $4 \%$ formaldehyde in PBS, washed in PBST (PBS with $0.1 \%$ Triton X-100) and PBSBT (PBST with $0.2 \%$ BSA) consecutively, and stained with the following antibodies in PBSBT: mouse $\alpha$-V5 (1:2000; Invitrogen) and rabbit $\alpha$ GFP (1:1000; Invitrogen). Secondary FITC-, Cy3-, or Cy5-conjugated antibodies were used at 
1:400 (Jackson Immunoresearch). To visualize F-actin, FITC- or Alexa 568-conjugated phalloidin (Invitrogen) was used at 1:500 in PBST.

\section{Mouse C2C12 myoblast culture}

A pair of predesigned siRNAs against the mouse Sptbn5 gene and negative control siRNA were obtained from Qiagen. RNAi was performed per manufacturer's instructions. Briefly, approximately $3 \times 10^{5}$ cells were seeded on each well of a 6 -well tissue culture dish and incubated overnight. On day 2 , cells were transfected with the mix of two siRNAs against Sptbn5 (200 pmol each) using HiPerFect transfection reagent (Qiagen). On day 3, the cells were washed and differentiated, and cells that were treated in parallel were subjected to RTPCR to access knock-down (KD) level. Fusion index was determined when the KD level was more than $80 \%$ as follows. Three days post-differentiation, cells were fixed and stained with anti-MHC antibodies (MF20) to identify differentiated cells and with CellMaskTM Orange membrane stain (Molecular Probes, Invitrogen) to visualize the overall cell/syncytia morphology. Cells were mounted using Prolong Gold antifade reagent with DAPI (Molecular Probes, Invitrogen) to visualize the nuclei. Ten random 40X microscopic fields under Axioscope 2 (Zeiss) were counted for each experiment and repeated in at least three independent experiments (total number of fields $\geq 30$ ). The fusion index was calculated as a mean percentage of nuclei in syncytia versus total number of nuclei per field in differentiated cells.

\section{Time lapse imaging and fluorescent recovery after photobleaching (FRAP)}

Time lapse imaging of embryos was performed as previously described ${ }^{4}$. Briefly, embryos expressing fluorescently tagged proteins in muscle cells were collected and dechorionated in $50 \%$ bleach. Subsequently, embryos were washed in water, placed onto a double-sided tape (3M), and covered with a layer of Halocarbon oil 700/27 (2:1; Sigma). Time lapse image acquisition was carried out on an LSM 700 Meta confocal microscope (Zeiss). 
The FRAP experiments were performed as previously described ${ }^{17}$. A region of interest (ROI) was manually selected and imaged in 3 to 5 frames to record the original fluorescent intensity (pre-bleach). Then the ROI was quickly bleached to a level at lower than $20 \%$ of its original fluorescent intensity and subsequently imaged with an appropriate time interval (post-bleach). The fluorescent intensities of the pre- and post-bleached ROI were measured using the image $\mathrm{J}$ program. The Prism software was used to determine the maximum recovery level (the percentage recovery compared to the pre-bleach level) and the half-time of recovery ( $\left.T_{\text {half }}\right)$ using a kinetic curve fit with an exponential decay equation.

\section{Structured illumination microscopy (SIM)}

Stage 13-14 embryos were fixed and stained as described above. The samples were then mounted in Prolong Gold (Molecular Probes) and imaged with an inverted microscope (Ti-E; Nikon) equipped with a 100x oil NA1.49 CFI SR Apochromat TIRF objective lens and an ORCAFlash 4.0 sCMOS camera (Hamamatsu Photonics K.K.). The images were acquired and processed with NIS-Elements AR software (Nikon).

\section{Electron microscopy}

Embryos were fixed by the high-pressure freezing and freeze substitution (HPF/FS) method as previously described $^{4,42}$. Briefly, a Bal-Tec device was used to freeze stage 12-14 embryos. Freeze-substitution was performed with $1 \%$ osmium tetroxide, $0.1 \%$ uranyl acetate in $98 \%$ acetone and $2 \%$ methanol on dry ice. Fixed embryos were embedded in Epon (Sigma-Aldrich) and cut into thin sections with an ultramicrotome (Ultracut R; Leica). The sections were mounted on copper grids and post-stained with $2 \%$ uranyl acetate for 10 min and Sato's lead solution ${ }^{43}$ for $1 \mathrm{~min}$ to improve image contrast. Images were acquired on a transmission electron microscope (CM120; Philips). 


\section{Recombinant protein purification and F-actin co-sedimentation assay}

To purify GST-fused $\beta_{H}$-Spec fragments from BL21-DE3 cells (NEB), protein expression was induced with $0.2 \mathrm{mM} \mathrm{IPTG}$ at room temperature for 12-15 hours. Cells were harvested and lysed by sonication in the lysis buffer: PBS (pH 7.4), 1\% Triton X-100, 5 mM DTT, 1 mM PMSF, and complete-mini protease inhibitor cocktail (Roche). After centrifugation, the supernatant was collected and incubated with pre-equilibrated glutathione-agarose resin at $4^{\circ} \mathrm{C}$ for $2-3$ hours. After washing in the lysis buffer, $\beta_{\mathrm{H}}$-Spec proteins were eluted with the Elution buffer: $50 \mathrm{mM}$ Tris (pH 7.5), $150 \mathrm{mM} \mathrm{NaCl}, 5 \mathrm{mM}$ DTT, $10 \mathrm{mM}$ glutathione (Sigma).

F-actin co-sedimentation assay was performed following the manufacturer's protocol (Cytoskeleton). Briefly, 0.5-1 $\mu \mathrm{M}$ purified proteins were incubated with $4 \mu \mathrm{M}$ F-actin assembled from monomeric actin for 1 hour in $\mathrm{F}$ buffer: $5 \mathrm{mM}$ Tris- $\mathrm{HCl}(\mathrm{pH} 8.0), 0.2 \mathrm{mM} \mathrm{CaCl}, 50 \mathrm{mM} \mathrm{KCl}$, $2 \mathrm{mM} \mathrm{MgCl} 2,1 \mathrm{mM}$ ATP. The F-actin/protein mixtures were centrifugated at $140,000 \mathrm{~g}$ for 30 min, and supernatants and pellets were separated and analyzed by SDS-PAGE and Coomassie Blue staining.

\section{Co-immunoprecipitation (Co-IP)}

Embryos expressing GFP-tagged Duf and Flag-tagged $\beta_{\mathrm{H}-S p e c}\left(k_{s} t^{\text {MIO3134-GFSTF.1 }}\right.$ /twi-GAL4;

UAS-Duf-GFP/+) were collected and dechorionated in $50 \%$ bleach. After being frozen in liquid nitrogen, embryos were disrupted in cold extraction buffer (50 mM Tris- $\mathrm{HCl}(\mathrm{pH} 8.5), 150 \mathrm{mM}$ $\mathrm{NaCl}, 0.5 \%$ sodium deoxycholate) with 20 strokes in a Dounce homogenizer. After centrifugation at $16,000 \mathrm{~g}$ for $20 \mathrm{~min}$, supernatants were removed and incubated with either anti-V5 (control) or anti-Flag $\left(\beta_{\mathrm{H}^{-}}\right.$Spec) antibodies at $4^{\circ} \mathrm{C}$ for $2-3$ hours. Protein-G-Sepharose beads (Roche) were used to precipitate antibodies. Precipitated proteins were analyzed by SDS-PAGE and western blotting. 


\section{Micropipette aspiration (MPA)}

Micropipette aspiration was performed as previously described ${ }^{44}$. Briefly, a pressure difference was generated by adjusting the height of a motor-driven water manometer. A fixed pressure of $0.4 \mathrm{nN} / \mu \mathrm{m}^{2}$ was applied instantly to the cell cortex of S2 cells with a polished glass pipette approximately 2-2.5 $\mu \mathrm{m}$ in radius. Images were collected in Schneider's medium supplemented with $10 \%$ FBS on an Olympus IX81 microscope with a 40x (1.3 NA) objective and a 1.6x optivar, utilizing MetaMorph software and analyzed using ImageJ. After background correction, the fluorescence intensity at the sites of protein accumulation was normalized against the opposite cortex of the cell $\left(\mathrm{I}_{\mathrm{b}} / \mathrm{I}_{\mathrm{O}}\right.$ or $\mathrm{I}_{\mathrm{t}} / \mathrm{I}_{\mathrm{O}}$, where $\mathrm{I}_{\mathrm{b}}, \mathrm{I}_{\mathrm{t}}$, and $\mathrm{I}_{\mathrm{o}}$ is the intensity in the cortex at the base, tip, or opposite side of the cell being aspirated). An ANOVA with Fisher's least significant difference was applied to determine statistical significance.

\section{Atomic force microscopy (AFM)}

$\mathrm{S} 2 \mathrm{R}+$ cells were plated on glass coverslips coated with concanavalin A (Sigma, St Louis, MO) and transfected to express fluorescently tagged Myoll and $\beta_{\mathrm{H}}$-Spec using Effectene (Qiagen), per the manufacturer's instructions. Lateral indentation experiments were conducted 2 days after transfection with a modified Catalyst AFM integrated with an Axio Observer fluorescence microscope (Zeiss). To determine the effect of a localized mechanical force on Myoll and $\beta_{\mathrm{H}^{-}}$ Spec localization, the cantilever (MLCT with a pyramidal tip, Bruker) was first brought into full contact, at around $50 \mathrm{nN}$ setpoint force, with the glass surface on a cell-free area within $10 \mu \mathrm{m}$ from a target cell. Next, the cell was laterally translated into the stationary cantilever using the piezoelectric XY stage and the NanoScope software (Bruker). The cantilever tip indented the edge of the cell by $2-5 \mu \mathrm{m}$. Cells were simultaneously imaged with a plan-apochromat $63 \times / 1.4$ NA oil immersion objective (Zeiss). Time lapse images were taken at 5 second intervals using the Micro-Manager software (http://micro-manager.org/wiki/Micro-Manager). 


\section{Coarse-grained molecular mechanics modeling}

In the coarse-grained model, the membrane-cortex composite is represented by a triangulated network where the nodes denote the crosslinking positions and the triangles resemble the meshes in the actin network, which is a network structure composed of proteins such as actin, actin crosslinkers, and Myoll. The system energy of the composite at the coarse-grained molecular level is calculated by

$$
E_{\text {system }}=E_{\text {bending }}+E_{\text {in-plane }}+E_{\text {sufface }}+E_{\text {volume }}
$$

where $E_{\text {bending }}$ is bending energy from the plasma membrane; $E_{\text {in-plane }}$ is the in-plane elastic energy associated with the deformation of actin crosslinkers and the dilation of each mesh; $E_{\text {surface }}$ is the surface energy of the whole cell; $E_{\text {volume }}$ is the energy associated with the volume conservation of the cell ${ }^{29,45,46}$. The motion of the nodes is calculated by the Brownian dynamics equation (or overdamped Langevin dynamics equation) where the driving force is the derivative of the system energy with respect to the local position.

The initial configuration of the system was thermally annealed at room temperature until the fluctuation of the system energy was negligible. This configuration was then mapped to the shape of a receiving cell. For simplicity, the protrusion has a cylindrical shape with the radius $r_{0}$ and length I on the surface of a cell with a diameter of $10 \mu \mathrm{m}$. The tip of the protrusion was a spherical cap of a radius of $r_{0}$. The final state of the system was achieved after $20 \mathrm{sec}$ of Brownian dynamics simulation with a time-step of $10^{-5} \mathrm{sec}$. The area dilation of each node was determined by averaging of the dilation of the triangles with which the node of interest was associated. The shear deformation of each node was calculated in a similar way. The contour of the deformation on the protrusion was plotted by the software Tecplot. The deformations along the length direction of the protrusion were obtained by the extraction tool of the software. 
37 Lee, S. K. \& Thomas, G. H. Rac1 modulation of the apical domain is negatively regulated by beta (Heavy)-spectrin. Mech Dev 128, 116-128 (2011).

38 Bulchand, S., Menon, S. D., George, S. E. \& Chia, W. The intracellular domain of Dumbfounded affects myoblast fusion efficiency and interacts with Rolling pebbles and Loner. PLoS One 5, e9374 (2010).

39 Kocherlakota, K. S., Wu, J. M., McDermott, J. \& Abmayr, S. M. Analysis of the cell adhesion molecule sticks-and-stones reveals multiple redundant functional domains, protein-interaction motifs and phosphorylated tyrosines that direct myoblast fusion in Drosophila melanogaster. Genetics 178, 1371-1383 (2008).

40 Byers, T. J., Brandin, E., Lue, R. A., Winograd, E. \& Branton, D. The complete sequence of Drosophila beta-spectrin reveals supra-motifs comprising eight 106-residue segments. Proc Natl Acad Sci U S A 89, 6187-6191 (1992).

41 Bour, B. A., Chakravarti, M., West, J. M. \& Abmayr, S. M. Drosophila SNS, a member of the immunoglobulin superfamily that is essential for myoblast fusion. Genes Dev 14, 1498-1511. (2000).

42 Zhang, S. \& Chen, E. H. in Cell Fusion: Overviews and Methods (ed E. H. Chen) 275297 (Humana Press, 2008).

43 Sato, T. A modified method for lead staining of thin sections. J Electron Microsc (Tokyo) 17, 158-159 (1968).

44 Kee, Y. S. \& Robinson, D. N. Micropipette aspiration for studying cellular mechanosensory responses and mechanics. Methods Mol Biol 983, 367-382 (2013).

45 Discher, D. E., Boal, D. H. \& Boey, S. K. Simulations of the erythrocyte cytoskeleton at large deformation. II. Micropipette aspiration. Biophys J 75, 1584-1597 (1998).

46 Li, J., Dao, M., Lim, C. T. \& Suresh, S. Spectrin-level modeling of the cytoskeleton and optical tweezers stretching of the erythrocyte. Biophys $\mathbf{J} \mathbf{8 8}, 3707-3719$ (2005). 

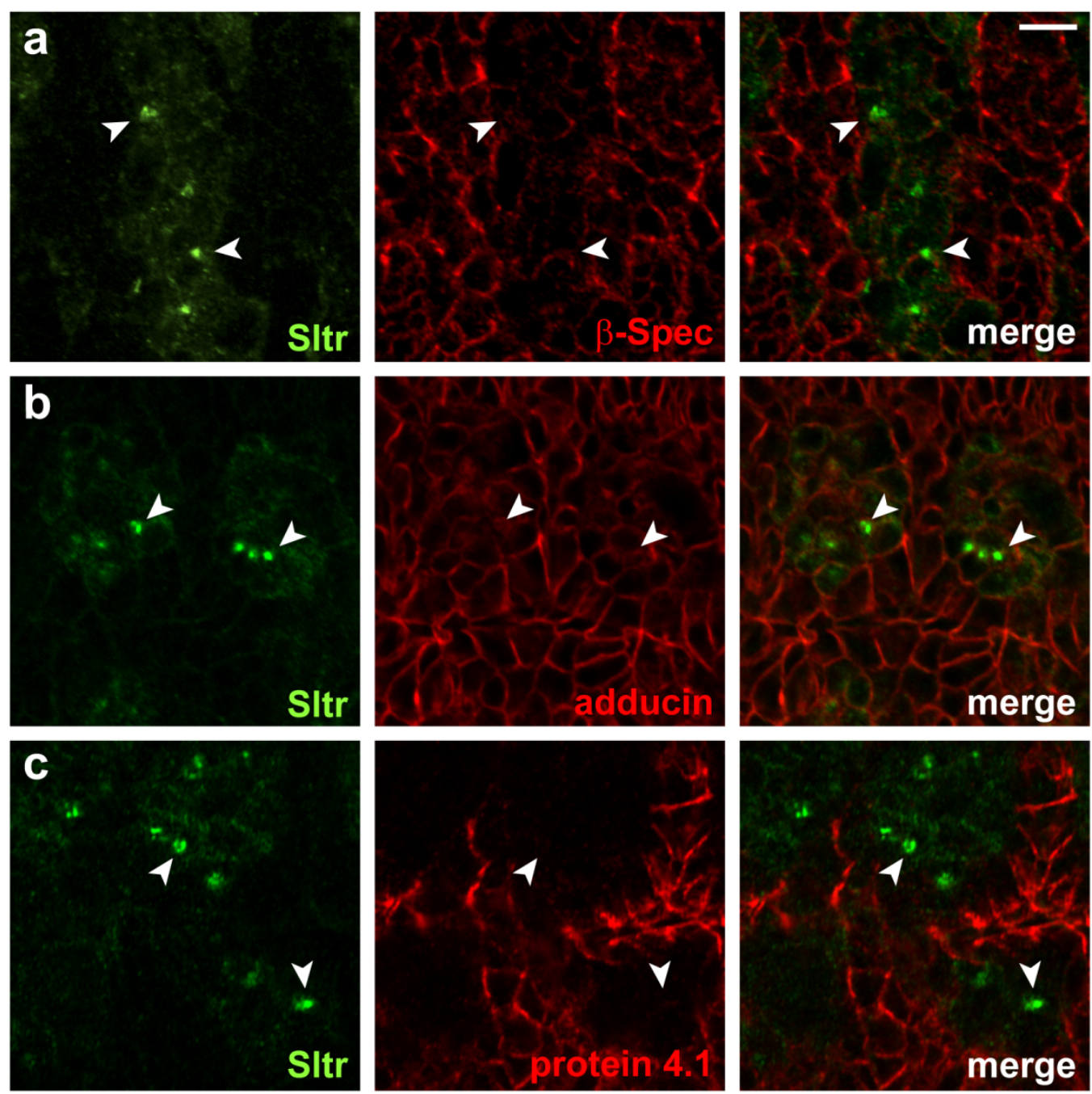

Extended Data Figure 1. $\beta$-spectrin, adducin and protein 4.1 are not enriched at the fusogenic synapse

Fusogenic synapses in stage 13-14 wild-type embryos were marked by anti-Sltr staining (green; arrowheads). $\beta$-Spec (a), adducin (known as hu-li tai shao in Drosophila) (b), and protein 4.1 (known as coracle in Drosophila) (c) were visualized by immunostaining with respective antibodies. Note that none of these proteins was enriched at the fusogenic synapse. Scale bar, $5 \mu \mathrm{m}$. 


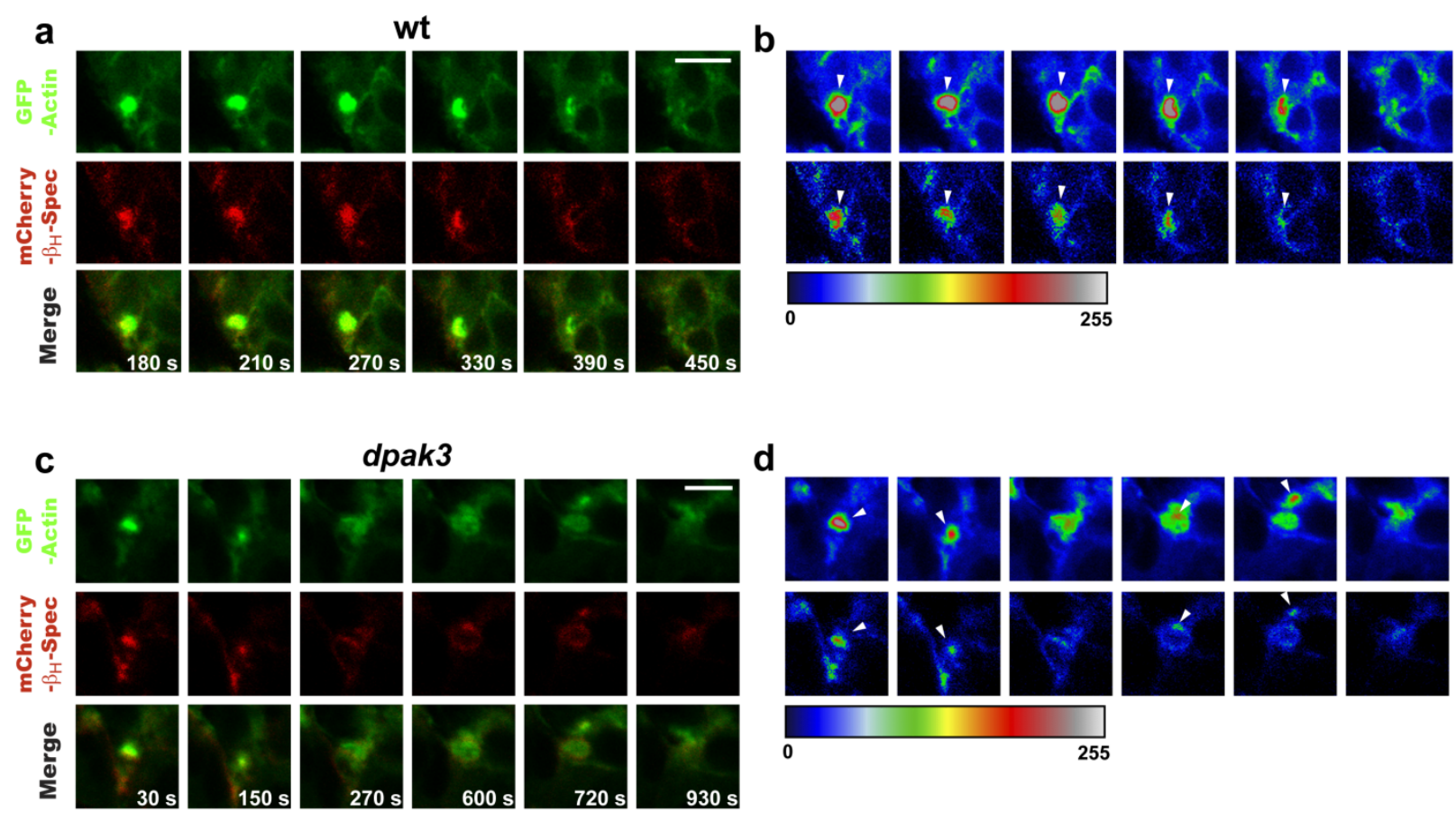

Extended Data Figure 2. Dynamic accumulation of spectrin at the fusogenic synapse in response to PLS invasion

Stills from time lapse movies of wt (a) or dpak3 mutant (c) embryos expressing GFP-actin (green) and mCherry- $\beta_{H^{-}}$Spec (red). The fluorescent intensities of F-actin foci and $\beta_{H^{-}}$Spec accumulation ( $\mathrm{a}$ and $\mathrm{c}$ ) were displayed by rainbow color-coded heat maps ( $b$ and $d$ ) on the arbitrary scale from 0 to 255 . F-actin in dpak3 mutant (c and d) was more diffused compared to the dense F-actin foci in wt ( $a$ and $b$ ). Note the dynamic changes in the intensity and morphology of $\beta_{\mathrm{H}}$-Spec accumulation correlating with the intensity of the F-actin foci (arrowheads in b and d). Scale bars, $5 \mu \mathrm{m}$. 
a

$\beta_{H}$-Spec

(4286aa)

$\beta_{H}-\operatorname{Spec} \Delta C$

$\beta_{H}-\operatorname{Spec} \Delta \mathbf{N}$

b

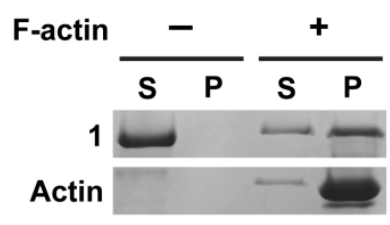

Actin-binding domain
Tetramerization site

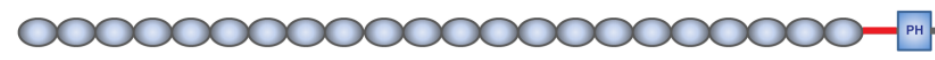

\section{Extended Data Figure 3. The $\mathrm{N}$-terminal $\mathrm{CH}$ domains of $\beta_{\mathrm{H}}$-spectrin bind F-actin}

(a) Domain structure of full length and mutant $\beta_{\mathrm{H}^{-}}$Spec used in MPA and F-actin cosedimentation assays. Actin-binding domains and the tetramerization site are indicated. Each distinct segment from the $\mathrm{N}$-terminus to the C-terminus of $\beta_{\mathrm{H}}-\mathrm{Spec}$ is designated by a number. $\mathrm{CH}$ : calponin homology; SH3: Src homology 3; $\mathrm{PH}$ : pleckstrin homology; Spec: spectrin repeat. (b) F-actin co-sedimentation assay with purified $\beta_{\mathrm{H}^{-}}$Spec fragments. The numbers $(1,29-31,34)$ indicate $\beta_{\mathrm{H}}$-Spec fragments depicted in (a). S: supernatant; P: pellet. Note that $\beta_{\mathrm{H}}-\mathrm{Spec}-1$ precipitated with F-actin, whereas $\beta_{\mathrm{H}}$-Spec-29-31 and $\beta_{\mathrm{H}}$-Spec-34 remained in the supernatant, confirming that the $\mathrm{CH}$ domains of $\beta_{\mathrm{H}^{-}}$Spec bind F-actin. 
a

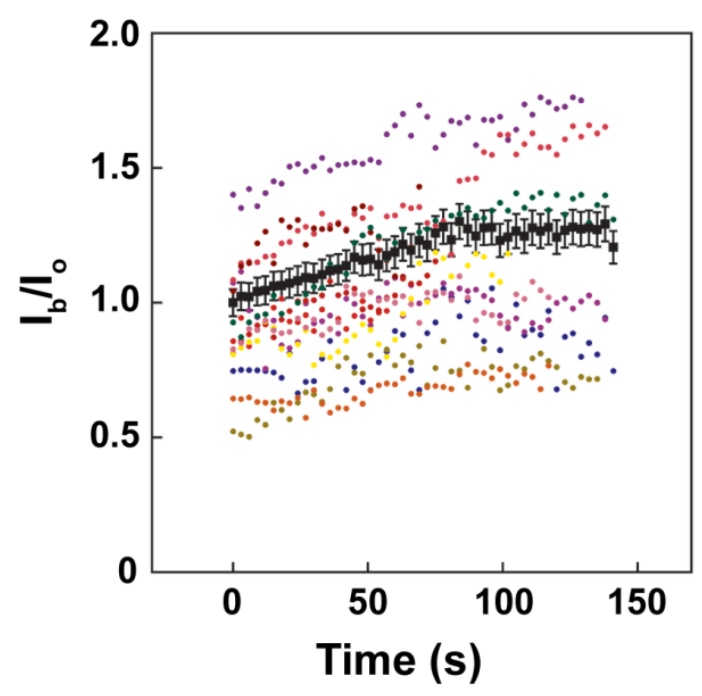

b

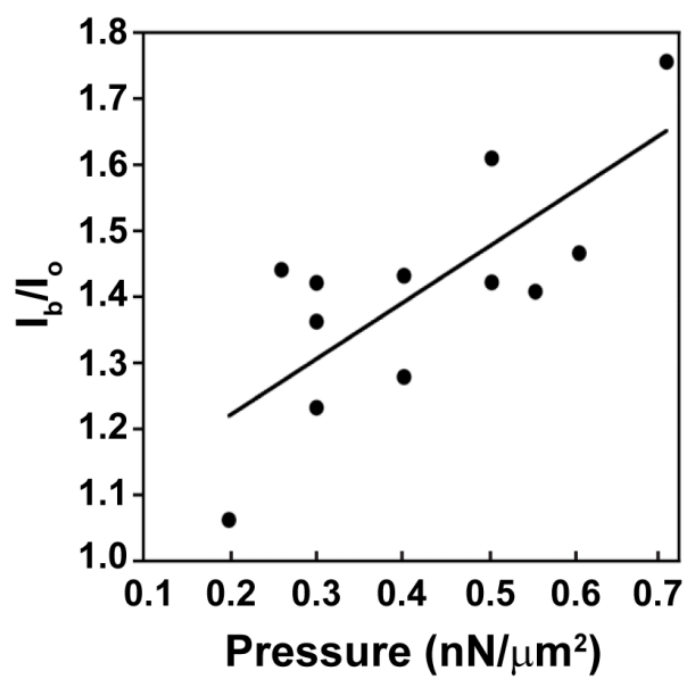

Extended Data Figure 4. The mechanosensitive accumulation of spectrin is time- and force-dependent

(a) $\beta_{\mathrm{H}}$-Spec accumulation over time in MPA experiments. Different color codes indicate traces of individual cells examined. The average values at each time point are shown as black dots with error bars (SEM). $\beta_{\mathrm{H}}$-Spec accumulation linearly increased over time and reached its peak at around $80-90 \mathrm{sec}$ after the onset of aspiration.

(b) $\beta_{\mathrm{H}^{-}}$Spec accumulation depends on applied force in MPA experiments. The increase of $\beta_{\mathrm{H}^{-}}$ Spec accumulation is proportional to the elevation of applied pressure. 


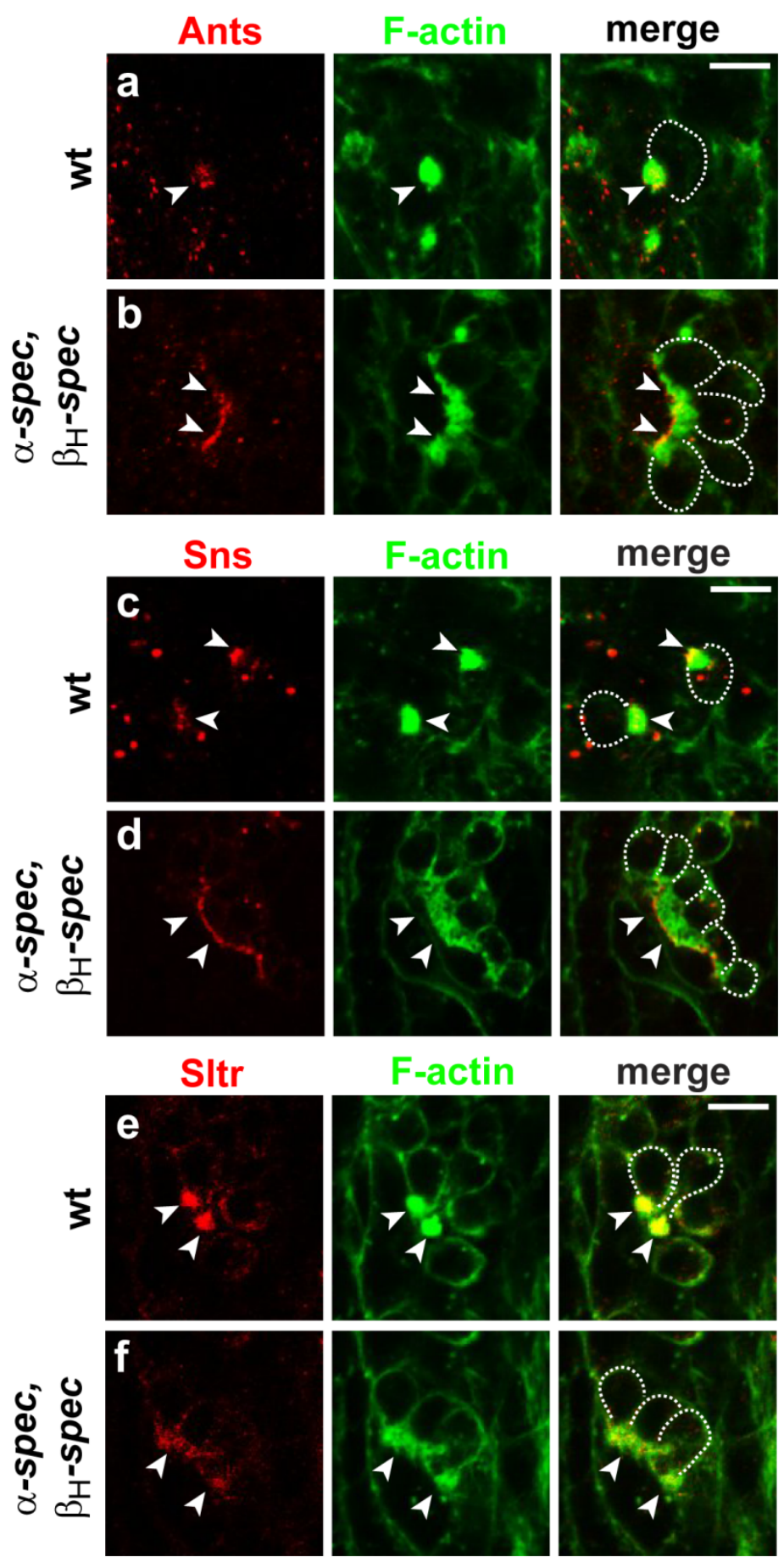

Extended Data Figure 5. Ants, Sns, SItr and F-actin are dispersed in $\alpha / \beta_{H^{-}}$spectrin double mutant embryos

Fusogenic synapses (arrowheads) in wt and $\alpha / \beta_{H^{-}}$spectrin mutant embryos were marked with Factin foci (phalloidin staining). The localization of Ants (a-b), Sns (c-d), and SItr (e-f) at the fusogenic synapse was visualized by immunostaining with respective antibodies. Note that all of 
these fusion-promoting proteins were dispersed in $\alpha / \beta_{H^{-}}$spectrin mutant embryos (b, $\mathrm{d}$, and $\mathrm{f}$, bottom left panels) compared to their focal aggregation in wt embryos (a, c and e, top left panels). FCMs are outlined in the merge panels. Scale bars, $5 \mu \mathrm{m}$. 


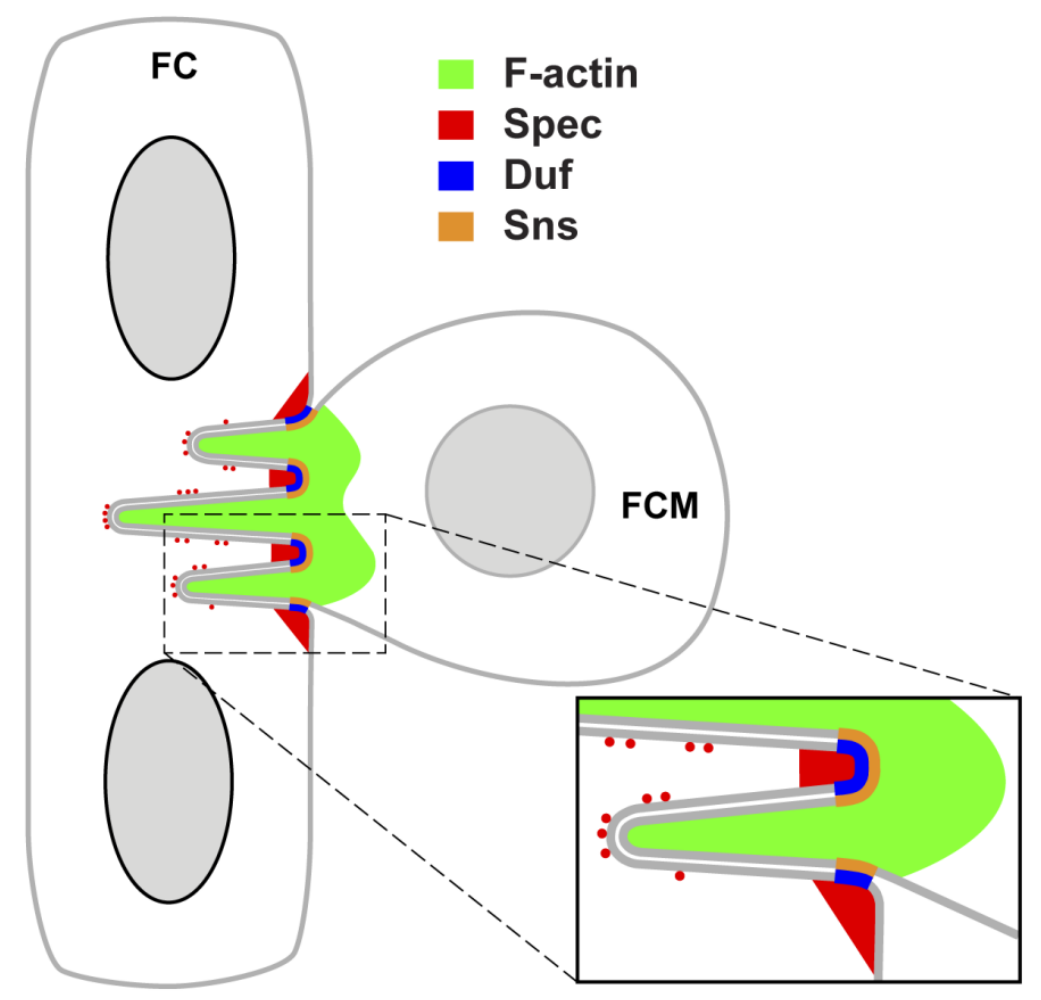

\section{Extended Data Figure 6. A working model of spectrin function at the fusogenic synapse}

The spectrin network in the founder cell functions as a "cellular sieve" to constrict invasive protrusions from the FCM. The mechanosensitive accumulation of spectrin (Spec; red) at the fusogenic synapse is triggered by pushing forces generated by the initial invasive protrusions from the FCM. The accumulated spectrin (1) restricts the cell adhesion molecules, Duf (founder cell; blue) and Sns (FCM; gold), to the fusogenic synapse via biochemical interactions; and (2) forms an unevenly spaced network containing spectrin-enriched and spectrin-free microdomains. Actin-propelled membrane protrusions (F-actin; green) can only squeeze through the spectrin-free, but not spectrin-enriched, microdomains. The resulting fingerlike protrusions continue to trigger mechanosensitive accumulation of spectrin (red dots in the receiving cell), which may constrict new protrusions. These long and thin protrusions put the fusogenic synapse under high local tension and promote plasma membrane fusion. 

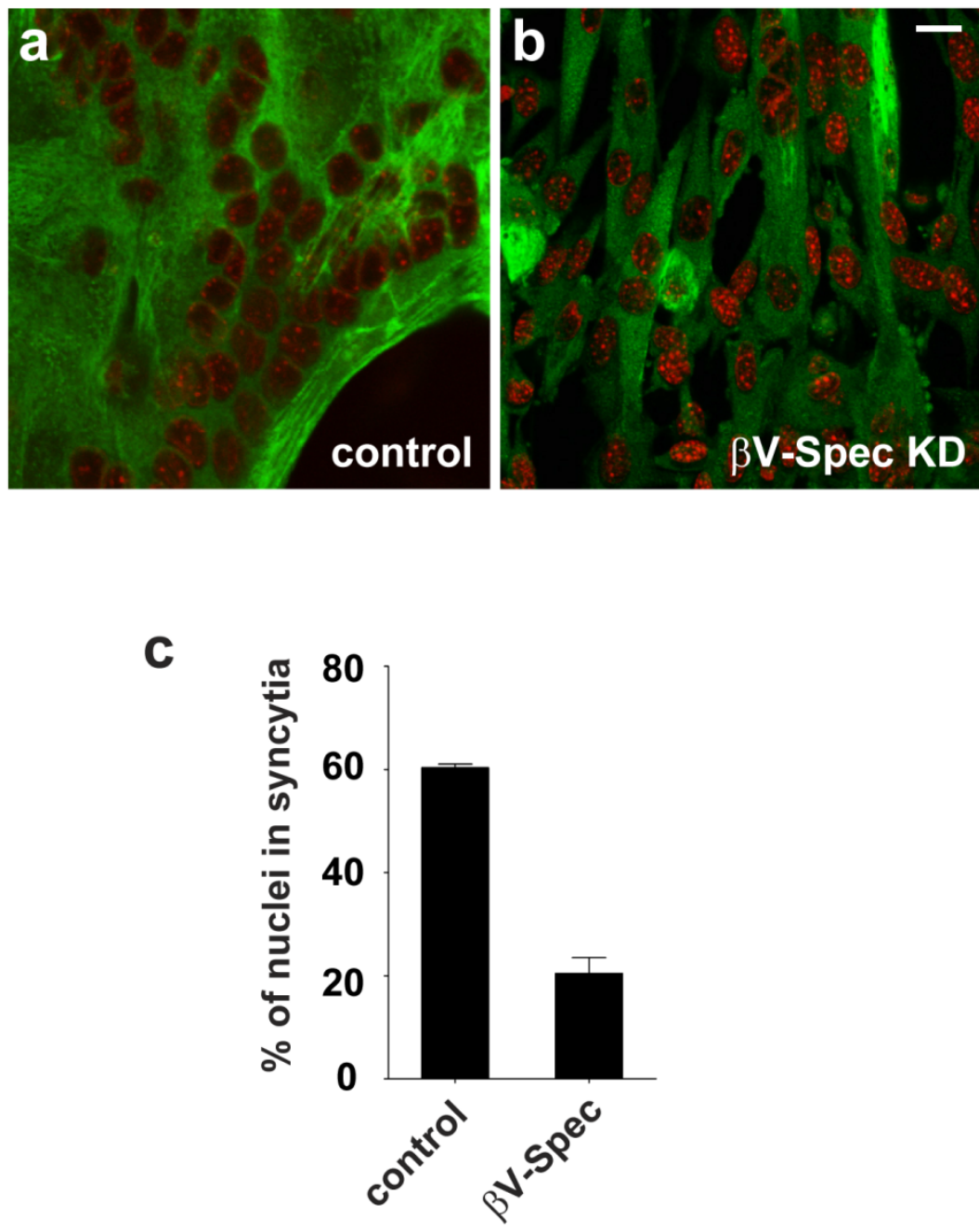

Extended Data Figure 7. $\beta V$-spectrin, the mammalian homologue of Drosophila $\beta_{\mathrm{H}^{-}}$ spectrin, is required for $\mathrm{C2C12}$ myoblast fusion

Confocal images of $\mathrm{C} 2 \mathrm{C} 12$ cells treated with either control siRNA (a) or siRNA directed against Sptbn5 ( $\beta$ V-spectrin) (b). Cells were allowed to differentiate for 72 hours, then fixed and stained with anti-MHC (green) and DAPI (red) to visualize differentiated muscle cells. (c) The fusion index was calculated as the percentage of the number of nuclei in multinucleated syncytia versus the total nuclei in differentiated muscle cells (MHC-positive). In each experiment, cells were counted in 10 random 40X microscopic fields and the data were averaged from three 
bioRxiv preprint doi: https://doi.org/10.1101/154831; this version posted June 23,2017 . The copyright holder for this preprint (which was not certified by peer review) is the author/funder. All rights reserved. No reuse allowed without permission.

independent experiments. Note that Sptbn5 knockdown (KD) significantly decreased the number of multinucleated syncytia. Error bar, standard deviation. Scale bar, $5 \mu \mathrm{m}$. 
Supplementary Video 1 . Spectrin is dynamically associated with the F-actin focus at the fusogenic synapse

Time-lapse imaging of muscle cells expressing GFP-actin (green) and mCherry- $\beta_{\mathrm{H}}$-spectrin (red) in a wild-type stage 14 embryo. The accumulation and dissolution of $\beta_{\mathrm{H}^{-}}$Spec coincided with the appearance and disappearance of the F-actin foci.

\section{Supplementary Video 2. Spectrin accumulation correlates with the F-actin foci intensity}

Time-lapse imaging of muscle cells expressing GFP-actin (green) and mCherry- $\beta_{\mathrm{H}}$-spectrin (red) in a stage 14 dpak3 mutant embryo. Arrow indicates a fusogenic synapse where the Factin focus underwent dynamic aggregation and dispersal. Note that the intensity of $\beta_{\mathrm{H}^{-}}$spectrin tightly correlated with that of the F-actin focus.

\section{Supplementary Video 3. Dynamic exchange of spectrin at the fusogenic synapse}

Fluorescent recovery after photobleaching (FRAP) of $\beta_{\mathrm{H}^{-}}$-spectrin at the fusogenic synapse (arrow). GFP-actin (green) and mCherry- $\beta_{\mathrm{H}}$-spectrin (red) were expressed in muscle cells in a stage 14 wild-type embryo. The mCherry fluorescence was photobleached and subsequently imaged every $30 \mathrm{sec}$. Note the rapid recovery of the mCherry signal after photobleaching.

\section{Supplementary Video 4. Mechanosensitive accumulation of spectrin and Myoll induced} by lateral indentation using AFM

Time-lapse imaging of an S2R+ cell co-expressing GFP- $\beta_{\mathrm{H}}$-spectrin (green) and RFP-Zipper (myosin heavy chain) (red). $\beta_{\mathrm{H}}$-spectrin and Myoll rapidly accumulated at the cell periphery

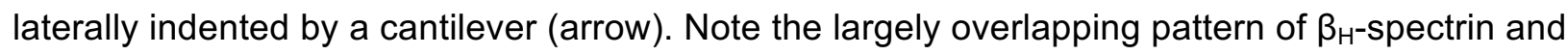
Myoll accumulation. 


\section{Supplementary Video 5. Duf dispersal at the fusogenic synapse in $\alpha / \beta_{H}$-spectrin double} mutant embryos

Time-lapse imaging of muscle cells expressing Duf-mCherry (red) and GFP-actin (green) in an $\alpha / \beta_{H^{-}}$spectrin mutant embryo. Duf initially aggregated to a small cluster associated with a dense F-actin focus (arrowhead), but gradually dispersed together with F-actin over time.

Supplementary Video 6. Duf resides in a tight cluster at the fusogenic synapse in wildtype embryos

Time-lapse imaging of muscle cells expressing mCherry-Duf (red) and GFP-actin (green) in a stage 14 wild-type embryo. Note that Duf remained in a tight cluster associated with each dense F-actin focus (arrowheads).

\section{Supplementary Video 7. F-actin dispersal in the absence of the cytoplasmic domain of}

\section{Duf}

Time-lapse imaging of muscle cells expressing mRFP-actin (red) and Duf $\Delta$ intra-Flag in a duf, rst mutant embryo. Note that F-actin initially formed a dense focus, but gradually dispersed over time. Arrowhead and arrow indicate two independent F-actin foci.

\section{Supplementary Video 8. 3D reconstruction of F-actin and spectrin at the fusogenic synapse based on SIM images \\ Embryo was labeled with F-actin (green) and $\beta_{\mathrm{H}-\mathrm{Spec}}$ (red). Note the mutually exclusive localization of $\mathrm{F}$-actin and $\beta_{\mathrm{H}^{-}}$Spec.}

